\title{
An Approach to Ship Deck Arrangement Optimization Problem Using an Improved Multiobjective Hybrid Genetic Algorithm
}

\author{
Hao Wang $(\mathbb{D})$ and Shunhuai Chen $(\mathbb{D})$ \\ Wuhan University of Technology, Wuhan, China \\ Correspondence should be addressed to Hao Wang; hao_wang@whut.edu.cn
}

Received 25 April 2021; Revised 8 July 2021; Accepted 16 August 2021; Published 29 August 2021

Academic Editor: Nunzio Salerno

Copyright (c) 2021 Hao Wang and Shunhuai Chen. This is an open access article distributed under the Creative Commons Attribution License, which permits unrestricted use, distribution, and reproduction in any medium, provided the original work is properly cited.

\begin{abstract}
Ship deck arrangement design is about determining the positions and dimensions of arranged objects. This paper presents the mathematical model for the ship deck arrangement optimization problem statement and how the individual's objective and constraint functions are computed. Moreover, an improved multiobjective hybrid genetic algorithm is redesigned to solve this complex nondeterministic problem and generate a set of diverse and rational deck arrangements in the early stage of ship design. An adaptive crossover operator and a novel topological replace operator invoked in this algorithm are described. Finally, the proposed algorithm is tested on a main deck arrangement optimization of an underwater detection ship. In the validation tests, the proposed algorithm is compared to the standard NSGA-II to determine its ability to produce a set of diverse and rational deck arrangements. Subsequently, the performance tests are used to determine the ability of the algorithm to work with the highly constrained arrangement problems and the efficiency of the adaptive crossover and topological replace operators.
\end{abstract}

\section{Introduction}

Ship deck arrangement design is about determining the positions and dimensions of arranged objects. Traditionally, different positions and dimensions of arranged objects have been manually adjusted until feasible arrangements emerge. As the size and complexity of ships increase, the design space becomes unprecedentedly large. Hence, this repetitive and manual method of trial-and-error might be limited, and manually generating arrangement designs can be a challenging and tedious task for humans. Computer algorithms that are being developed to generate desired designs under given design objectives and constraints show enormous potential in expediting the design process and improving the design quality.

The computer-aided algorithms of arrangement design in ships have been applied since the late 1970s [1]. For comparison and measurement of both rigid and flexible goals and constraints, the Fuzzy Set Theory was firstly used to assess the ship arrangements in 1985 [2]. The ship arrangement design involves multiple goals and constraints and an overwhelming number of design variables, thus finding the optimal arrangement appears prohibitive. However, numerous attempts and various optimization methodologies have been used over the years to solve the ship arrangement optimization problem. The related methodologies vary from genetic algorithms and expert systems to multiple-criteria decision-making techniques and network science theory.

The suitable breakdown of arrangements is a commonly adopted strategy to reduce the difficulty of solving the ship general arrangement optimization problem. Daniels and Parsons et al. [3-6] broke down the ship general arrangement problem into dozens of Zone-deck arrangement problems (where a Zone-deck was divided by a deck and the major structural boundaries in a subdivision). After the optimal allocation of arranged objects to Zone-decks was determined, the ship arrangement optimization was simplified to a lot of Zone-deck arrangement optimizations. And a hybrid agent-genetic algorithm was proposed for solving the Zone-deck arrangement optimization. Lee and Han et al. $[7,8]$ proposed an improved genetic algorithm 
applied to the ship compartment arrangement, in which the inner structure bulkheads and passages were considered. Similarly, the ship general arrangement design might consist of dozens of compartment arrangement designs. In their research, an adjacency graph was used to represent the relationships between arranged objects and a rectilinear distance method was used to calculate the distance between two arranged objects.

Boulougouris et al. [9] simplified the ro-ro ship arrangement problem into an allocation problem and used the genetic algorithm to solve it. Olçer $[10,11]$ integrated multiple-objective optimization with a fuzzy multiattribute group decision-making technique to solve the subdivision optimization of ro-ro ships. In these cases, part of the geometric information was ignored to reduce the difficulty of solving, so it is impossible to generate specific geometric arrangements, but rather some decisions and recommendations of the ship general arrangement design. In 2013, a network theory-based method using nonspatial networks was introduced to ship arrangement design by Gillespie et al. [12-14]. All geometric information of the arranged objects and the ship was completely ignored. Although no concrete arrangement was generated by this method, the rudimentary design knowledge obtained by that was ideally suited to the early stage arrangement design.

Van Oers and Wangers et al. [15-19] proposed a 3D packing approach to solve the ship general arrangement optimization, in which all arranged objects were considered at the same time for numerous potential alternatives. Although a nondominated sorting genetic algorithm II (NSGA-II) was used in this approach, the arrangement optimization was not a complete optimization. A single objective, named packing density, was used by the NSGA-II to generate relatively dense ship arrangements. As the implementation of NSGA-II required at least two objectives, a constant function was provided as a second. It might mean that the hundreds of ship arrangements generated by the NSGA-II were the only feasible arrangements at the physical level. To determine the optimal arrangements, the designers would decide after a thorough comparison of the hundreds of feasible ship arrangements based on their experience and knowledge.

Beyond the field of ships, there is much research being done on computer-aided algorithms for arrangement design. Kim and Roh et al. [20-22] developed an arrangement method based on a multistage optimization strategy and an expert system to assist in the arrangement design of submarines. As stated in their papers, solving the submarine arrangement optimization by considering all arranged objects at the same time presented untenable difficulties. A three-stage optimization consisting of compartment arrangement optimization, subcompartment arrangement optimization, and element arrangement optimization was used. In the field of architecture, the genetic algorithm is still a common method. Thakur and Kumari et al. [23] used a genetic algorithm to generate the topological arrangement for a single flat of apartments with regular-shaped spaces. As the size of the arrangement space was smaller and the number of rooms was fewer, the specific geometric arrangements could be generated from the topological arrangement. Rodrigues et al. [24-26] presented a singleobjective optimization model aggregated by seven design requirements to describe the arrangement design in architecture. And a hybrid evolutionary algorithm was proposed to solve this optimization problem. Rodrigues et al. [27] also applied the hybrid evolutionary algorithm to the optimization of thermal performance in the urban layout design. And the annealed neural network was applied to assist the architectural layout design in paper [28]. In addition, there is some research on the layout design algorithm of a room, where the scale of the optimization is relatively small. Liang et al. [29] introduced the game theory to the cabin layout design. A noncooperative game was formulated to assist the designer in achieving as high of a performance as possible in a cabin layout design. To assist the designer in generating an optimal room layout in a reasonable time, Bénabès et al. [30] developed an interactive optimization strategy and Inoue and Takagi [31] proposed a room layout algorithm with its underlying rules.

The heuristic algorithms have better performance in solving the arrangement optimization problems in the field of architecture. With further expansion of the optimization problems, these algorithms are not effective enough. The literature review indicates that solving the ship arrangement optimization by considering all arranged objects at the same time presents untenable difficulties. The complexity of ship general arrangement optimization is clearly beyond the limitation of these existing algorithms. Therefore, in the previous studies, the ship arrangement optimization problems are usually decomposed or simplified into dozens of subproblems to accommodate the capabilities of the existing algorithms. The dimensions of the arrangement space in these subproblems are much smaller than that of the ship deck, and the number of the arranged objects arranged in these subproblems is limited.

The remainder of this paper is organized as follows. Section 2 describes the ship deck arrangement problem and the nomenclature and formulation of the multiobjective mathematical model. Section 3 presents an improved multiobjective hybrid genetic algorithm (IMOHGA). In Section 4, the deck arrangement optimization of an underwater detecting ship is conducted and the computation results are presented. The computation results, the advantages, and the limitations of this approach are discussed in Section 5. Finally, the conclusion of this paper is made in Section 6.

\section{Mathematical Model}

General arrangement design is the initial link of ship design. Ship deck arrangement design is an important part of general arrangement design. The multiple layers of interconnections and dependencies among arranged objects, which are not immediately discernible, make it impossible to directly determine the positions of all arranged objects based on prior knowledge. The traditional ship deck arrangement design in the early stage strongly depends on the ship designer's experience and intuition, which is a highly subjective 
and ambiguous spiral process. When the ship arrangements are generated automatically by a computer algorithm, the evaluation criterion for arrangements and the method to tackle the overlaps are significant. In other words, the objective functions and constraints are two important aspects in guiding the algorithm to find rational arrangements.

2.1. Problem Statement. Ship deck arrangement design in early stages can be stated as determining the dimensions of a set of predetermined objects $\mathbf{E}=\left\{E_{1}, E_{2}, \ldots, E_{N}\right\}$ and arranging them on deck space $D$. The nomenclatures for the formulation of the deck arrangement optimization problem are listed in Table 1. Since the fore and aft parts of the ship deck are usually equipped with specific deck machines (e.g., the anchor windlass is arranged on the bow of the main deck), the influence of the fore and aft parts of the deck shape on the arrangement can be ignored in the early stage of ship design. In this paper, the deck space $D$ is simplified as a rectangle plane, where the length is equal to the ship length $L$ and the width is equal to the ship breadth $B$. The arranged objects are considered as cuboids with five parameters, two positional parameters $(x$ and $y$ ) and three-dimensional parameters (length $l$, width $w$, and height $h$ ). In the deck arrangement design, all cabins have to fit the deck height, and some deck machines have specific heights. In order to finally display the 3D layout result, it is necessary to define the height of the arranged objects. The position of the arranged objects is determined by the reference point $(x, y)$, which is the vertex point of the cuboids near the starboard quarter, as shown in Figure 1.

Compared with the design variables of the width $w$ and length $l$ of arranged objects, their height $h$ may have less impact on the single-deck arrangement. And to reduce the number of design variables, the height $h$ must not be determined as design variables in the optimization. However, the height $h$ of arranged objects would be used for the display of the three-dimensional deck arrangements. By consequence, there are two types of design variables to be used in the optimization, namely, positional variables $(x$ and $y)$ and dimensional variables $(l$ and $w$ ). The design variables are expressed in equation (1), where $n$ signifies the number of arranged objects. The ship deck arrangement optimization problem can be formulated as equations (2)-(4). This mathematical model is composed of three objective functions (topological objective function $F_{1}$, geometric objective function $F_{2}$, and overlapping objective function $F_{3}$ ) and two sets of constraint functions (position constraint functions $\vec{C}_{\text {position }}$ and overlapping constraint functions $\vec{C}_{\text {overlap }}$ ), the details of which are described in the next two sections.

$$
\begin{aligned}
& \vec{v}=\left(x_{1}, \ldots, x_{n}, y_{1}, \ldots, y_{n}, w_{1}, \ldots, w_{n}, l_{1}, \ldots, l_{n}\right), \\
& Z: \min \vec{F}(\vec{v})=\left[F_{1}(\vec{v}), F_{2}(\vec{v}), F_{3}(\vec{v})\right]^{T}, \\
& \text { s.t. } \vec{C}_{\text {position }}(\vec{v})<0, \\
& \text { s.t. } \vec{C}_{\text {overlap }}(\vec{v})<0 .
\end{aligned}
$$

TABLE 1: The nomenclature for the formulation of the deck arrangement optimization problem.

\begin{tabular}{lc}
\hline & Nomenclature \\
\hline$E_{i}$ & Arranged object $i$ \\
$n$ & The number of arranged objects \\
$B$ & Ship breadth \\
$f_{c}, f_{s}$ & Adjacency and separation evaluators \\
$F_{i}$ & Objective function \\
$\omega_{c}, \omega_{s}$ & Weight coefficients of evaluators $f_{c}$ and $f_{s}$ \\
$\theta_{c}$ & Normalized coefficient \\
$\mathrm{Mc}$ & Material flow cost matrix \\
$\mathrm{Us} s_{i j}$ & Separation satisfaction of $E_{i}$ and $E_{j}$ \\
$d c_{i j}$ & Connection distance of $E_{i}$ and $E_{j}$ \\
$d x_{i j}$ & Interval distance of $E_{i}$ and $E_{j}$ on $x$ direction \\
$s_{i}$ & Area of $E_{i}$ \\
$S_{\text {deck }}$ & Area of deck space \\
$p_{i j}$ & The length of bulkheads shared by $E_{i}$ and $E_{j}$ \\
$\mathbf{E}$ & The set of arranged objects \\
$D$ & Deck space \\
$L$ & Ship length \\
$f_{d}, f_{b}$ & Compactness and bulkheads utilization evaluators \\
$C$ & Constraints set \\
$\omega_{d}, \omega_{b}$ & Weight coefficients of evaluators $f_{d}$ and $f_{b}$ \\
$\mathrm{Mf}$ & Material flow frequency matrix \\
$\mathrm{Mr}$ & Separation relationship matrix \\
$\mathrm{U} \mathrm{o}_{i j}$ & Overlapping of $E_{i}$ and $E_{j}$ \\
$d s_{i j}$ & Separation distance of $E_{i}$ and $E_{j}$ \\
$d y_{i j}$ & Overlapping area of $E_{i}$ and $E_{j}$ \\
$s_{o i j}$ & Deck utilization \\
$u_{d}$ &
\end{tabular}

2.2. Objective Function. The ship deck arrangement optimization using computer algorithms is an evolving process with the purpose of satisfying the design requirements and improving the design quality. A number of design goals (deck utilization, habitability, efficiency of operations, etc.) need to be considered in the ship arrangement optimization, most of which are fuzzy and implicit [12]. In manual arrangement design, it is sufficient for designers to roughly estimate these fuzzy and implicit goals based on their domain knowledge and experience. The difficulty of using a computer to evaluate the design goals is that multiple layers of objectives may be determined by secondary and tertiary effects that are not immediately discernible. It is necessary to rationally simplify the objective functions. Inspired by the topological relationship network generated by the Intelligent Ship Arrangement (ISA) [13], a topological objective function and a geometric objective function are defined in this paper. Two relative-location-based topological relations (adjacency and separation) are used to calculate the topological objective function, and two utilizations (deck area utilization and bulkhead utilization) are used to calculate the geometrical objective function. In addition, an overlapping objective function different from the above two objective functions is introduced to accelerate convergence.

The topological objective function $F_{1}$ aggregated by the adjacency evaluator $f_{c}$ and the separation evaluator $f_{s}$ is used to evaluate the topology relationship between 


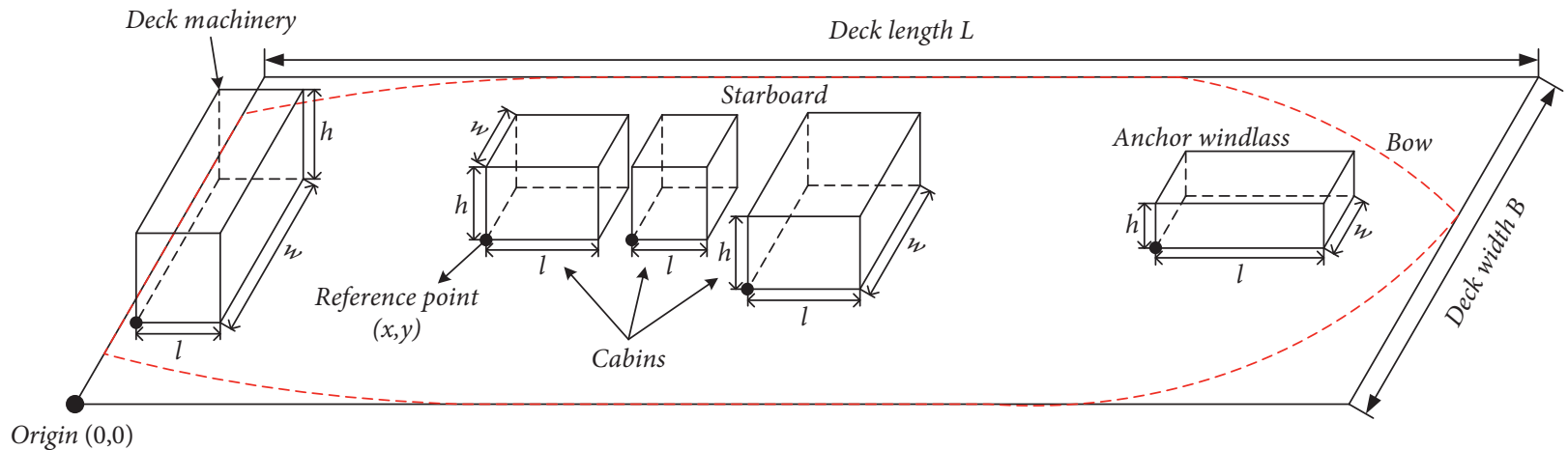

FIgURE 1: Geometrical representation of ship deck arrangement.

arranged objects, as expressed in equation (5). The values of the weight coefficients $\omega_{c}$ and $\omega_{s}$ are determined by designers according to the importance of the separation and adjacency. And the sum of $\omega_{c}$ and $\omega_{s}$ must be equal to 1 .

$$
F_{1}=\omega_{c} \cdot f_{c}+\omega_{s} \cdot f_{s} .
$$

The adjacency evaluator $f_{c}$ expressed by equation (6) assesses the adjacency satisfaction based on the material flow frequency matrix $\mathrm{Mf}$ and the material flow cost matrix Mc. Considering that the adjacency evaluator $f_{c}$ is not a normalized expression, a normalized coefficient $\theta_{c}$ is introduced to make it normalized. However, since the upper bound of the adjacency satisfaction is hard to accurately estimate, the evaluator $f_{c}$ is only approximately normalized. The material flow frequency $\mathrm{Mf}_{i j}$ represents the number of trips to be made between arranged objects $E_{i}$ and $E_{j}$ in one period, and the material flow cost $\mathrm{Mc}_{i j}$ represents the cost per unit distance traveled between arranged objects $E_{i}$ and $E_{j}$. For example, in one day, there are usually three trips between the crew cabin and the mess (breakfast, lunch, and dinner). The material flow cost per unit distance traveled between the crew cabin and the mess is related to the number of crews on these trips. The ship designers could predetermine the material flow frequency and cost according to the design preference. In consequence, the adjacency satisfaction between the two elements $E_{i}$ and $E_{j}$ is completely dependent on their connection distance $d c_{i j}$ in the optimization. Accurately calculating the practical connection distance $d c_{i j}$ during the early stage design appears prohibitive. In this paper, the connectivity distances are simplified in a rectilinear form and calculated by equation (7). If there is an overlap between $E_{i}$ and $E_{j}$, the connectivity distance $d c_{i j}$ is equal to the ship length $L$ as a penalty; if there is no overlap, the connectivity distance $d c_{i j}$ would be the sum of the $x$ direction distance $d x_{i j}$ calculated by equation (8) and $y$ direction distance $d y_{i j}$ calculated by equation (9). As it is shown in Figure 2, the distances $d x_{i j}$ and $d y_{i j}$ are the interval distances of arranged objects $E_{i}$ and $E_{j}$ in $x$ and $y$ directions.

$$
\begin{aligned}
f_{c} & =\theta_{c} \cdot \sum_{i=1}^{n-1} \sum_{j=i+1}^{n}\left(d c_{i j} \times \mathrm{Mf}_{i j} \times \mathrm{Mc}_{i j}\right), \\
d c_{i j} & = \begin{cases}L, & \text { if } d x_{i j}<0 \cap d y_{i j}<0, \\
d x_{i j}+d y_{i j}, & \text { if } d x_{i j} \geq 0 \cap d y_{i j} \geq 0, \\
d x_{i j}, & \text { if } d x_{i j} \geq 0 \cap d y_{i j}<0, \\
d y_{i j}, & \text { if } d x_{i j}<0 \cap d y_{i j} \geq 0,\end{cases} \\
d x_{i j} & =\max \left\{\left(x_{i}+l_{i}\right),\left(x_{j}+l_{j}\right)\right\}-\min \left\{x_{i}, x_{j}\right\}-\left(l_{i}+l_{j}\right),
\end{aligned}
$$

The separation evaluator $f_{s}$ expressed by equation (10) assesses the separation satisfaction Us according to the predetermined separation relationship matrix Mr. The separation evaluator $f_{s}$ is composed of a minimum value of the separation satisfaction and a weighted average of the separation satisfaction. The minimum of the matrix Us represents the worst separation satisfaction between arranged objects. And the weighted average is the average of the separation satisfactions between the arranged objects with separation requirements. The global average of all separation satisfactions cannot reflect the actual level of separation satisfaction, because the separation satisfaction between the arranged objects without separation requirements is considered as fully acceptable and equal to 1 . This may result in an overestimation of the separation satisfaction. By this way, the separation evaluator can seek to raise the average level of separation satisfaction while also ensuring that the worst separation satisfaction is acceptable. The square root is used to make this evaluator normalized and "one minus" is for minimizing optimization. The matrix element $\mathrm{Mr}_{i j}$ is used to determine whether there is a separation requirement between two arranged objects $E_{i}$ and $E_{j}$. If the value of $\mathrm{Mr}_{i j}$ is 1 , the arranged object $E_{i}$ should be arranged separately from $E_{j}$; if it is 0 , it means that there is no separation requirement between $E_{i}$ and $E_{j}$. For the two 


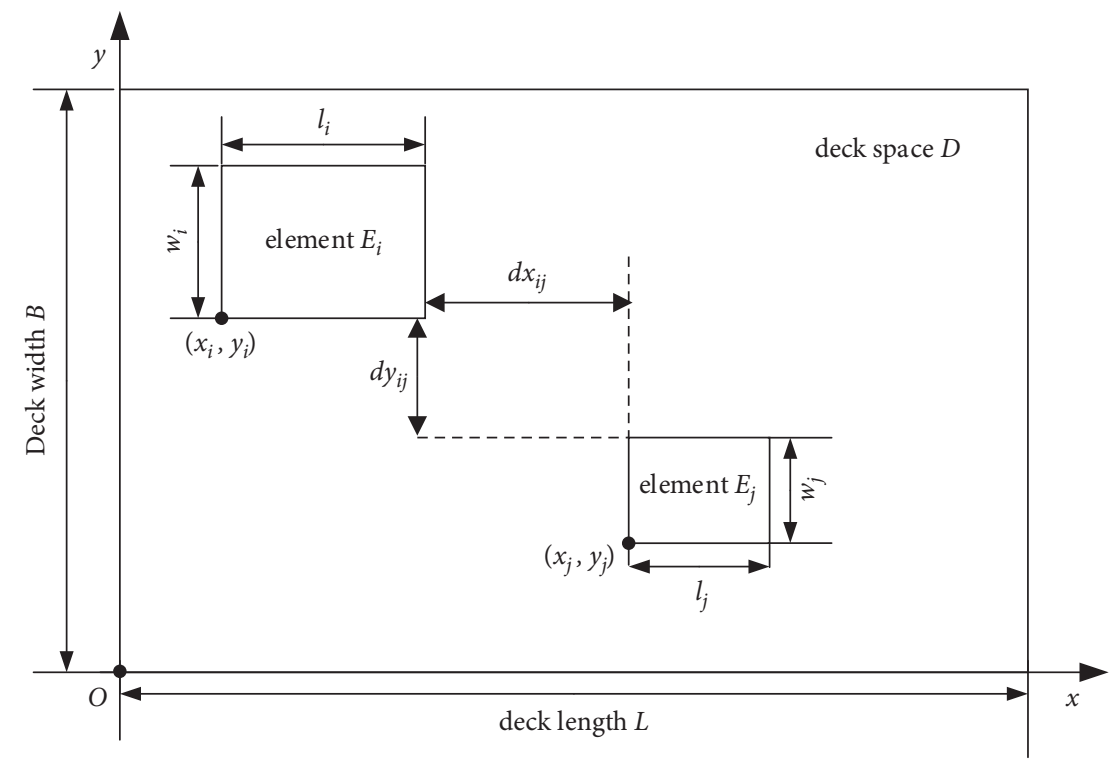

Figure 2: Interval distances of elements $E_{i}$ and $E_{j}$ in $x$ and $y$ directions.

arranged objects with a separation requirement, the separation satisfaction $\mathrm{Us}_{i j}$ depends on the separation distance $d s_{i j}$. However, the separation satisfaction between arranged objects is commonly quite subjective. Fuzzy Set Theory may be an ideal way to deal with this type of problem. A fuzzy membership function expressed by equation (11) is introduced to calculate the separation satisfaction $\mathrm{Us}_{i j}$ between two arranged objects $E_{i}$ and $E_{j}$, as shown in Figure 3. The region with $\mathrm{Us}_{i j}=0$, where the separation distance $d s_{i j}$ is smaller than the threshold $\lambda_{1}$, means clearly unacceptable, and region with $U s_{i j}=1$, where the separation distance $d s_{i j}$ exceeds the separate goal $\lambda_{2}$, means fully acceptable. The fuzzy region between the threshold $\lambda_{1}$ and the separate goal $\lambda_{2}$ is a fuzzy and subjective quantity between 0 and 1 . The values of $\lambda_{1}$ and $\lambda_{2}$ are predetermined by designers. The separation distance $d s_{i j}$ can be calculated by equation (12), which is similar to the calculation of the connectivity distance $d c_{i j}$. The difference is that when two arranged objects are overlapping, the distance $d s_{i j}$ would be equal to 0 instead of the ship length $L$, and if the space distances $d x_{i j}$ and $d y_{i j}$ are bigger than 0 , the separation distance $d s_{i j}$ would be the linear distance between two elements $E_{i}$ and $E_{j}$.

$$
\begin{gathered}
f_{s}=1-\sqrt{\min (\mathrm{Us}) \cdot \frac{\sum_{i=1}^{n} \sum_{j=1}^{n}\left(\mathrm{Us}_{i j} \cdot \mathrm{Mr}_{i j}\right)}{\sum_{i=1}^{n} \sum_{j=1}^{n} \mathrm{Mr}_{i j}},} \\
\mathrm{Us}_{i j}= \begin{cases}1, & \text { if } \mathrm{Mr}_{i j}=0, \\
0, & \text { if } \mathrm{Mr}_{i j}=1 \cap d s_{i j} \leq \lambda_{1}, \\
\frac{1}{\lambda_{2}-\lambda_{1}} \cdot d s_{i j}+\frac{\lambda_{1}}{\lambda_{1}-\lambda_{2}}, & \text { if } \mathrm{Mr}_{i j}=1 \cap \lambda_{1}<d s_{i j} \leq \lambda_{2}, \\
1, & \text { if } \mathrm{Mr}_{i j}=1 \cap d s_{i j}>\lambda_{2},\end{cases}
\end{gathered}
$$

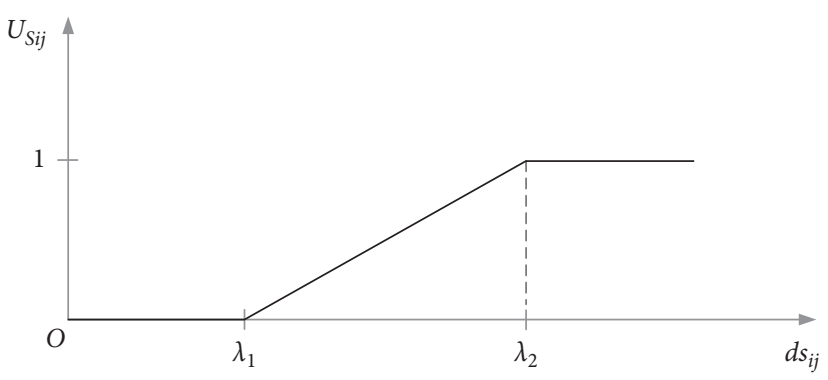

FIGURE 3: Fuzzy membership function of the separation satisfaction $\mathrm{Us}_{i j}$.

$$
d s_{i j}= \begin{cases}0, & \text { if } d x_{i j}<0 \cap d y_{i j}<0, \\ \sqrt[2]{d x_{i j}^{2}+d y_{i j}^{2}}, & \text { if } d x_{i j} \geq 0 \cap d y_{i j} \geq 0, \\ d x_{i j}, & \text { if } d x_{i j} \geq 0 \cap d y_{i j}<0, \\ d y_{i j}, & \text { if } d x_{i j}<0 \cap d y_{i j} \geq 0 .\end{cases}
$$

The geometric objective function $F_{2}$ aggregated by the compactness evaluator $f_{o}$ and the bulkhead utilization evaluator $f_{w}$ is used to evaluate the geometric quality of the deck arrangements, as expressed in equation (13). Similarly, the values of the weight coefficients $w_{w}$ and $w_{o}$ are predetermined by designers according to the importance of compactness and bulkhead utilization. And the sum of $w_{o}$ and $w_{w}$ must be equal to 1 .

$$
F_{2}=\omega_{d} \cdot f_{d}+\omega_{b} \cdot f_{b}
$$

The compactness evaluator $f_{d}$ expressed by equation (14) assesses the compactness of the ship arrangement based on the deck utilization $U_{d}$. The deck utilization $U_{d}$ is calculated by equation (15), where $s_{i}$ signifies the area of the arranged object $E_{i}$ and $S_{\text {deck }}$ signifies the area of deck space. The compactness evaluator is a fuzzy membership function 
defined using two halves of Normal Distribution, as shown in Figure 4. The parameter $\mu$ is the best deck utilization defined by designers. When the deck utilization $U_{d}$ is equal to the best deck utilization $\mu$, the compactness of the ship arrangement reaches the optimal level, and the compactness evaluator $f_{o}$ would be equal to 0 . Typically, the tolerance for the deck space with excess area is more relaxed than the crowded arrangement. Hence, designers can select different $\sigma_{1}$ and $\sigma_{2}$, where the value of $\sigma_{2}$ is usually smaller than $\sigma_{1}$.

$$
\begin{aligned}
& f_{d}= \begin{cases}1-\frac{\left(1 /\left(\sqrt{2 \pi} \cdot \sigma_{1}\right) e^{-\left(u_{d}-\mu\right)^{2} / 2 \sigma_{1}^{2}}\right)}{\left(1 /\left(\sqrt{2 \pi} \cdot \sigma_{1}\right)\right)}, & \text { if } a \leq \mu, \\
1-\frac{\left(1 /\left(\sqrt{2 \pi} \cdot \sigma_{2}\right) e^{-\left(u_{d}-\mu\right)^{2} / 2 \sigma_{2}^{2}}\right)}{\left(1 /\left(\sqrt{2 \pi} \cdot \sigma_{2}\right)\right)}, & \text { if } a>\mu,\end{cases} \\
& u_{d}=\frac{\sum_{i=1}^{N}\left(s_{i}\right)}{S_{\text {deck }}} .
\end{aligned}
$$

The bulkhead utilization evaluator $f_{b}$ expressed by equation (16) is used to make the ship arrangements closer in practice, instead of merely rectangles adjacent to each other. All arranged objects are considered surrounded by four bulkheads in this paper. Due to the presence of some bulkheads shared by two arranged objects, the total length of bulkheads is usually less than the sum of the perimeter of all arranged objects. The length $p_{i j}$ of bulkheads shared by two elements $E_{i}$ and $E_{j}$ can be calculated by equation (17). To improve the utilization efficiency of the bulkheads and the rationality of the deck arrangement, it can be assumed that the smaller the total length of bulkheads is, the better the arrangement design would be. The sharing of bulkheads can be divided into $x$ and $y$ directions, and they cannot occur simultaneously.

$$
\begin{gathered}
f_{b}=\frac{\sum_{i=1}^{n}\left[2 \cdot\left(l_{i}+w_{i}\right)\right]-\sum_{i=1}^{n-1} \sum_{j=1}^{n} p_{i j}}{\sum_{i=1}^{N}\left[2 \cdot\left(l_{i}+w_{i}\right)\right]}, \\
p_{i j}= \begin{cases}-d x_{i j}, & \text { if } d x_{i j}<0 \cap d y_{i j}=0, \\
-d y_{i j}, & \text { if } d x_{i j}=0 \cap d y_{i j}<0 .\end{cases}
\end{gathered}
$$

The overlapping objective function $F_{3}$ calculated by equations (18)-(20) measures the degree of the overlap in the deck arrangement using the average of overlap areas, where $s_{o i j}$ signifies the overlap area of arranged objects $E_{i}$ and $E_{j}$ and $\mathrm{Uo}_{i j}$ is used to determine whether $E_{i}$ and $E_{j}$ overlap. If $E_{i}$ and $E_{j}$ overlap, $\mathrm{Uo}_{i j}$ is equal to 1 ; otherwise, $\mathrm{Uo}_{i j}$ is equal to 0 . No overlap would be allowed in the deck arrangement, which means that the optimal value of this objective must be zero. Although the arrangements with less overlap can be generated under the guidance of the overlapping objective function, it is difficult to find the arrangements without any overlap. The requirement of no overlapping is actually a type of constraint, and a set of complete and rigorous constraint functions is critical to ensure that this arrangement requirement is met. By consequence, the main purpose of this

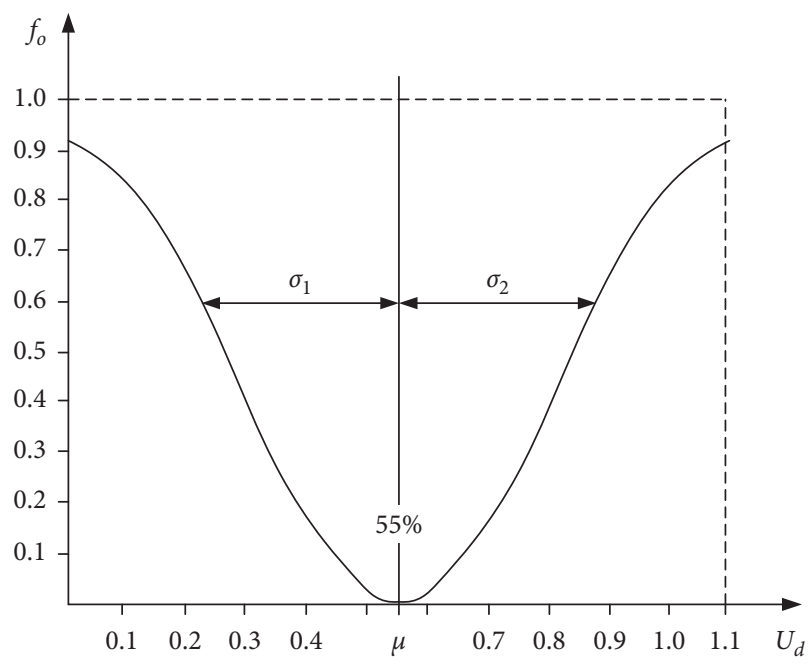

FIgURE 4: Fuzzy membership function of the deck utilization $U_{d}$.

objective function is used to compare the degree of the overlap between the solutions with the same number of overlapping constraint violations.

$$
\begin{aligned}
F_{3} & =\frac{\sum_{i=1}^{n-1} \sum_{j=i+1}^{n} s_{o i j}}{\sum_{i=1}^{n-1} \sum_{j=i+1}^{n} U_{o i j}}, \\
s_{o i j} & = \begin{cases}d x_{i j} \cdot d y_{i j}, & \text { if } d x_{i j}<0 \cap d y_{i j}<0, \\
0, & \text { otherwise, }\end{cases} \\
U o_{i j} & = \begin{cases}1, & \text { if } d x_{i j}<0 \cap d y_{i j}=0, \\
0, & \text { otherwise. }\end{cases}
\end{aligned}
$$

2.3. Constraint Functions. In the arrangement optimization, the constraints are mainly used to ensure the feasibility of the arrangement at the physical level. Therefore, the constraint functions are composed of the basic geometric requirements of the ship deck arrangement design in this paper. According to the mathematical forms of these functions, the constraints can be divided into two categories: linear constraints and nonlinear constraints.

The linear constraint functions are used to ensure that the arranged objects do not exceed the boundaries of the deck space. Since the positional variable $x$ is determined in the feasible domain $[0, L]$ and the positional variable $y$ is determined in the feasible domain $[0, B]$, the front and (or) left sides of the arranged object may exceed the boundaries of the deck space. The dimensions of arranged objects are also uncertain in the optimization, so it is hard to fully satisfy these geometric requirements by modifying the feasible domains of position variables. Two linear constraint functions expressed in equations (21) and (22) are used to ensure this arrangement requirement.

$$
\begin{gathered}
x_{i}+l_{j}-L<0, \\
y_{i}+w_{j}-B<0 .
\end{gathered}
$$


Generating deck arrangements without overlap is a critical and challenging part of the arrangement optimization based on computer algorithms. If and only if the projections of the arranged objects $E_{i}$ and $E_{j}$ on $x$ - and $y$-axes overlap at the same time, they would overlap in the deck space. The overlapping constraints of the projections on $x$ - and $y$-axes between $E_{i}$ and $E_{j}$ could be expressed by equations (23) and (24). Therefore, the overlapping constraints in the deck space used to eliminate overlaps are nonlinear equality constraints and could be expressed by equation (25).

$$
\begin{aligned}
p_{x i j} & =\left(x_{i}+l_{i}-x_{j}\right) \cdot\left(x_{i}-x_{j}-l_{j}\right)-\left|\left(x_{i}+l_{i}-x_{j}\right) \cdot\left(x_{i}-x_{j}-l_{j}\right)\right|, \\
p_{y i j} & =\left(y_{i}+w_{i}-y_{j}\right) \cdot\left(y_{i}-y_{j}-w_{j}\right)-\left|\left(y_{i}+w_{i}-y_{j}\right) \cdot\left(y_{i}-y_{j}-y_{j}\right)\right|, \\
p_{x i j} \cdot p_{y i j} & =0 .
\end{aligned}
$$

\section{Solution Methodology}

3.1. Multiobjective Hybrid Genetic Algorithm. The ship deck arrangement optimization problem proves to be a nondeterministic polynomial hard problem [32], which means that it is unsolvable in polynomial time, so many optimization algorithms are based on heuristics. Furthermore, due to multidesign objectives that need to be considered in the optimization, problem-solving methods are concentrated on finding a Pareto-optimal front. However, since the ship arrangement design demonstrates complex nonlinear characteristics in its constraints, it is difficult to solve a largescale ship arrangement problem by the traditional heuristic algorithms. For a ship arrangement optimization problem with $n$ arranged objects, the number of decision variables may be $4 n$ and the number of nonlinear constraints is $((n-1) n / 2)$. The literature review shows that, in the previous studies, the ship arrangement optimization problems are solved by decomposing it into dozens of subproblems, where the arrangement space is much smaller than the ship deck. In this paper, an IMOHGA is redesigned to solve the ship deck arrangement problem. However, finding mathematically optimal solutions appears prohibitive for the proposed algorithm. Instead, it is proposed to find diverse solutions that satisfy all constraints as well as possible to approximate the true Pareto-front. Figure 5 depicts the flowchart of the improved multiobjective hybrid genetic algorithm. This algorithm that couples an improved multiobjective genetic algorithm with a local stochastic search strategy can be generalized in three steps:

Step 1. Initial stage: firstly, the algorithm randomly generates the individuals according to the input data. The input data used in this algorithm is listed in Table 2, including the specified feasible domains of the design variables and the size of deck space. Although the feasible domains of the positional variables $x$ and $y$ can be directly determined by the arrangement space, the designers may define more rigorous and accurate feasible domains for some special arranged objects, such as anchor gears and cranes. Due to the fact that the requirements for the dimension of arranged objects in ship arrangement design are usually given in terms of area $s$, it is difficult for designers to directly define the feasible domains of the dimensional variables $l$ and $w$. In consequence, the feasible domains of the area $s$ and aspect ratio $r$ of arranged objects may be selected as the input data for the convenience of the designers. The specified value of the area $s_{i}$ and aspect ratio $r_{i}$ of an arranged object $E_{i}$ can be randomly determined within their feasible domains and its length $l_{i}$ and width $w_{i}$ can be calculated by equations (26) and (27). To ensure that the arranged objects do not exceed the boundaries of the deck space, the randomly generated individuals are required to meet the linear geometric constraints. The positional variable $x_{i}$ needs to be rerandomly generated in the new feasible domain $\left[0, L-l_{i}\right]$ if the front end of the arranged object $E_{i}$ is beyond the deck space $\left(x_{i}+l_{i}>L\right)$, and the positional variable $y_{i}$ needs to be rerandomly generated in the new feasible domain $\left[0, B-w_{i}\right]$ if the left side of the arranged object $E_{i}$ is beyond the deck space $\left(y_{i}+w_{i}>B\right)$. Finally, an initial population $P_{0}$ with a size of $N$ is formulated.

$$
\begin{aligned}
l_{i} & =\sqrt{s_{i} \cdot r_{i}}, \\
w_{i} & =\sqrt{\frac{s_{i}}{r_{i}}}
\end{aligned}
$$

Step 2. Global search stage: an improved multiobjective genetic algorithm is used for the global search. Firstly, all individuals in the initial population $P_{0}$ are evaluated according to the objective functions and sorted using the modified definition of domination [33]. The tournament selection operator is used to determine the parental individuals that would have to be used to generate new individuals, and an adaptive crossover operator and a random mutation operator are used to generate new individuals. The adaptive crossover operator is designed based on the characteristics of the ship deck arrangement optimization, which can increase the dispersion of the population and avoid damaging good patterns in the parents. Afterwards, these new individuals would be incorporated into the parent population $P_{i}$ to form an intermediate population $P_{i}^{\prime}$. With the purpose of maintaining the scale 


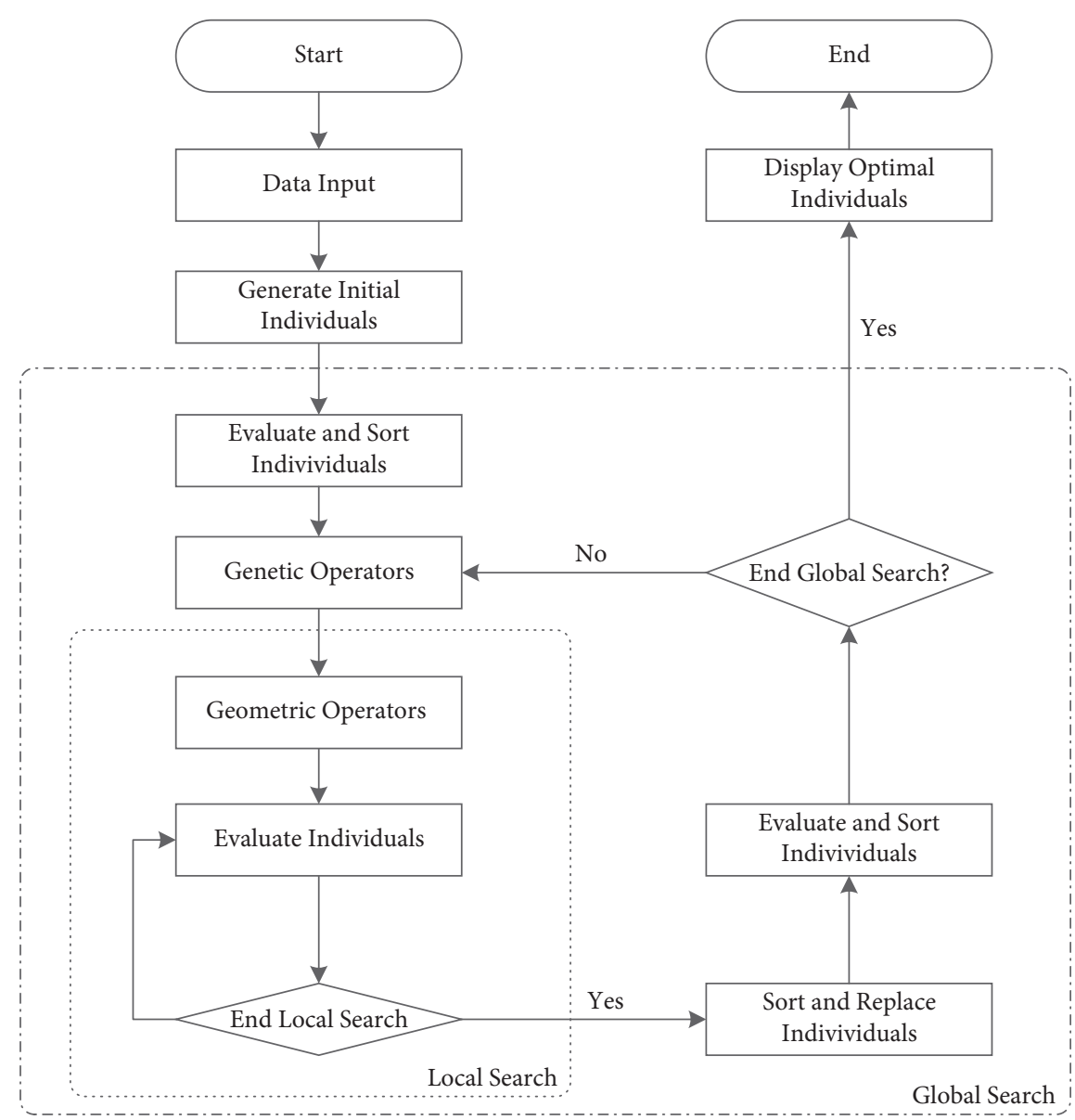

FIgURE 5: The improved multiobjective hybrid genetic algorithm flowchart.

TABle 2: The list of input data for the ship deck arrangement optimization.

The feasible domains of position variables

The feasible domains of the area of arranged objects

The feasible domains of the aspect ratio of arranged objects

Material flow frequency Mf

Material flow cost Mc

Separation relationship $\mathrm{Mr}$

The length of deck space $L \quad 47$

Normalized coefficient $\theta_{c} \quad 0.05$

Separation threshold $\lambda_{1}$

Similar zone parameter $\lambda_{t} \quad 0.5$

Overlapping threshold $\lambda_{o}$

Standard deviation $\sigma_{2} \quad 0.2$

Adaptive probability $p_{1} \quad 0.85$

Adaptive probability $p_{3} \quad 0.25$

Population size

Input data

\begin{tabular}{cc} 
The length of deck space $B$ & 12.2 \\
Coefficients $\omega_{c}, \omega_{s}, \omega_{d}, \omega_{b}$ & 0.5 \\
Separation goal $\lambda_{2}$ & 7 \\
Replacement threshold $\lambda_{r}$ & 0.45 \\
Best deck utilization $\mu$ & 0.55 \\
Standard deviation $\sigma_{1}$ & 0.25 \\
Adaptive probability $p_{2}$ & 0.5 \\
Crossover probability $p_{c}$ & 0.85 \\
Maximum iterations & 300 \\
\hline
\end{tabular} and diversity of the population, an elitist strategy and a novel topological replace operator are applied to the intermediate population $P_{i}^{\prime}$ to generate an offspring population $P_{i+1}$ with a size of $N$. The details of the adaptive crossover and topological replace operators are presented in the next two sections.
Step 3. Local search stage: during this stage, the individuals are subject to a set of stochastic geometric operators that perform geometric transformation on the phenotype level of the individuals [34]. The geometric operators only apply to the position variables without affecting the dimension variables. They are 
intended to find the local optimal individual around the individual generated by the genetic operators. Although the new individual generated by the geometric operators may be similar to its parent individual, the geometric operators have a good effect on the improvement of the overlapping objective $F_{3}$ and the bulkhead utilization $f_{b}$. Therefore, the local search can improve the ability of the algorithm to search feasible solutions and make the generated arrangements closer in practice, instead of merely rectangles adjacent to each other.

3.2. Crossover Operators. Crossover operators are an important part of the genetic algorithm. The crossover operators are used to keep good patterns in individuals as much as possible and increase the dispersion of the population. However, the crossover operators would not be able to keep their excellent performance for generating the new individuals in the arrangement optimization. In this subsection, two classical crossover operators (multiple-point crossover; simulated binary crossover) are discussed, and an adaptive crossover operator is proposed.

3.2.1. Multiple-Point Crossover. In the arrangement optimization problem, the multiple-point crossover operators can be divided into two types according to the crossover objects. The first type of multiple-point crossover operator is applied to the arranged object level. Part of the arranged objects would be selected as the crossover objects, and all design variables of the crossover objects would be exchanged between two individuals at the same time. The second type of multiple-point crossover operator is applied to the level of design variables. The design variables would be independently determined whether to be exchanged between two individuals. Since no new variable value is produced using the two types of multiple-point crossover operators, the search space is limited. For a population of size $N$, each design variable has at most $N$ possible values, which are determined by the initial population. It is assumed that the design variables for everyone in the initial population are different. Therefore, no matter how many generations have evolved, the newly generated individuals are always at the $N^{4}$ mesh points determined by the initial population. Hence, it is not appropriate to use the multiple-point crossover operator in the arrangement optimization.

3.2.2. Simulated Binary Crossover. The simulated binary crossover is a typical representative of the real-coded crossover operator, which applies the mechanism of the binary-based single-point crossover to the real-coded chromosomes. The two children $\overrightarrow{c_{1}}$ and $\overrightarrow{c_{2}}$ generated by the parent individuals $\overrightarrow{v_{1}}=\left(v_{1}^{1}, v_{2}^{1}, \ldots, v_{4 n}^{1}\right)$ and $\overrightarrow{v_{2}}=\left(v_{1}^{2}, v_{2}^{2}, \ldots, v_{4 n}^{2}\right)$ using the simulated binary crossover operator could be calculated by equation (28). And a distribution function defined by the designers is used in the simulated binary crossover to ensure the random generation of children, as shown in equation (29). In this equation, $u_{i}$ is a random number in the interval $(0,1)$ and $\delta$ is the dispersion exponent defined by designers. It can be observed that the simulated binary crossover pays close attention to the search of each independent design variable and neglects the coupling relationships of design variables. Thus, new values of design variables could be produced, but the offspring individuals cannot inherit the good patterns from the parental individuals. This means that, by using the simulated binary crossover operator, two solutions satisfying all constraints would result in two solutions that violate lots of constraints.

$$
\begin{gathered}
\left\{\begin{array}{l}
c_{i}^{1}=0.5 \cdot\left[\left(1+\beta_{i}\right) \cdot v_{i}^{1}+\left(1-\beta_{i}\right) \cdot v_{i}^{2}\right], \\
c_{i}^{2}=0.5 \cdot\left[\left(1-\beta_{i}\right) \cdot v_{i}^{1}+\left(1+\beta_{i}\right) \cdot v_{i}^{2}\right],
\end{array}\right. \\
\beta_{i}=\left\{\begin{array}{cl}
\left(2 u_{i}\right)^{1 /(\delta+1)}, & 0<u_{i} \leq 0.5, \\
\frac{1}{2\left(1-u_{i}\right)}{ }^{1 /(\delta+1)}, & 0.5<u_{i} \leq 1 .
\end{array}\right.
\end{gathered}
$$

3.2.3. Adaptive Crossover. With the purpose of keeping good patterns and increasing the dispersion of the population, an adaptive crossover operator that couples the multiple-point crossover with the simulated binary crossover is proposed and used in the global search process. The global search could be divided into three phases according to the minimum number $c_{\text {min }}$ of overlapping constraint violations of individuals in the population. And a violation threshold $\varepsilon_{\max }$ defined by designers is introduced to determine the global search phase.

If the minimum overlapping constraint violation $c_{\min }$ is greater than the violation threshold $\lambda_{o}$, the global search can be considered in the first phase. Since the initial population is randomly generated, there are many violations of the overlapping constraints in the individual in the first phase. It could be considered that there is no good pattern in the individuals and the primary purpose of the crossover operator is to quickly find new individuals with less violation of overlapping constraints in this phase. The simulated binary crossover may be an effective crossover operator. The drawback of simulated binary crossover leading to incoherent arrangements (solutions with lots of constraint violations) is negligible because all arrangements in the population are incoherent. Therefore, the simulated binary crossover would be invoked with a high probability $p_{1}$ and the multiple-point crossover would be invoked with a low probability $\left(1-p_{1}\right)$ in the first phase of the global search. And the multiple-point crossover operator is applied to the level of design variables.

After the first stage, the number of overlapping constraint violations in the individual has been rapidly reduced and stabilized at a lower level. When the minimum overlapping constraint violation $c_{\text {min }}$ is reduced to less than the violation threshold $\lambda_{o}$ but greater than 0 , the global search would enter the second phase. In this phase, good patterns have been generated in the population and the primary 
purpose of the crossover operator is to find the individuals without overlap and increase the dispersion of the population. To keep the good patterns and further generate new individuals, the simulated binary crossover and multiplepoint crossover would invoke with equal probability $p_{2}=50 \%$. And the multiple-point crossover operator is applied to the level of design variables in this search phase.

When a feasible individual is generated in the population, the minimum number $c_{\min }$ of the overlapping constraint violations of individuals in the population would be equal to 0 and the global search would enter the last phase. The feasible individuals in this paper represent the individuals who satisfy all overlapping constraints. In this phase, the primary purpose of the crossover operator transforms to generate more new feasible individuals and ensure the diversity of the population. Therefore, the good patterns in the population should be kept as much as possible, the simulated binary crossover would be invoked with a low probability $p_{3}$, and the multiple-point crossover would be invoked with a high probability $\left(1-p_{3}\right)$. The multiple-point crossover operator is applied to the level of the arranged objects in this search phase.

3.3. Topological Replace Operator. In the ship arrangement optimization based on computer algorithms, the criterion for judging whether the two individuals are similar is crucial. The judgment about whether the two arrangements are similar is a subjective and fuzzy task. Unlike conventional optimization problems, the arrangement optimization emphasizes the difference of design variables rather than the objective functions. Since a uniformly spread out Paretooptimal front cannot guarantee the diversity of the arrangements, the crowding distance method of maintaining the diversity in the multiobjective genetic algorithm is useless here. To efficiently make consistent judgments and ensure the diversity of population, a topological replace operator is designed.

As mentioned above, two categories of design variables, dimensional variables $(l$ and $w$ ) and positional variables $(x$ and $y$ ), are used to determine the arranged objects. However, the influence of dimensional and positional variables on the similarity of ship deck arrangements is significantly different.

The dimensional variables $(l$ and $w)$ are limited by the area and aspect ratio of the arranged objects. Figure 6 shows the transformation (the top-right vertex point of the arranged object can only change in the shaded zone) of an arranged object within the limited range of area and aspect ratio. The minimum area $S_{\min }$ of arranged objects is limited by some ship design standards, such as $<$ Maritime Labor Convention, 2006>, and the max area $S_{\min }$ of arranged objects could be determined by design requirements and experience. Ship designers decided the range of aspect ratio according to the internal layout. The area of deck space is usually hundreds of times the area of arranged objects. Therefore, the difference in ship deck arrangement caused by the dimension of arranged objects is indistinctive and can be ignored in the early stage of ship design.

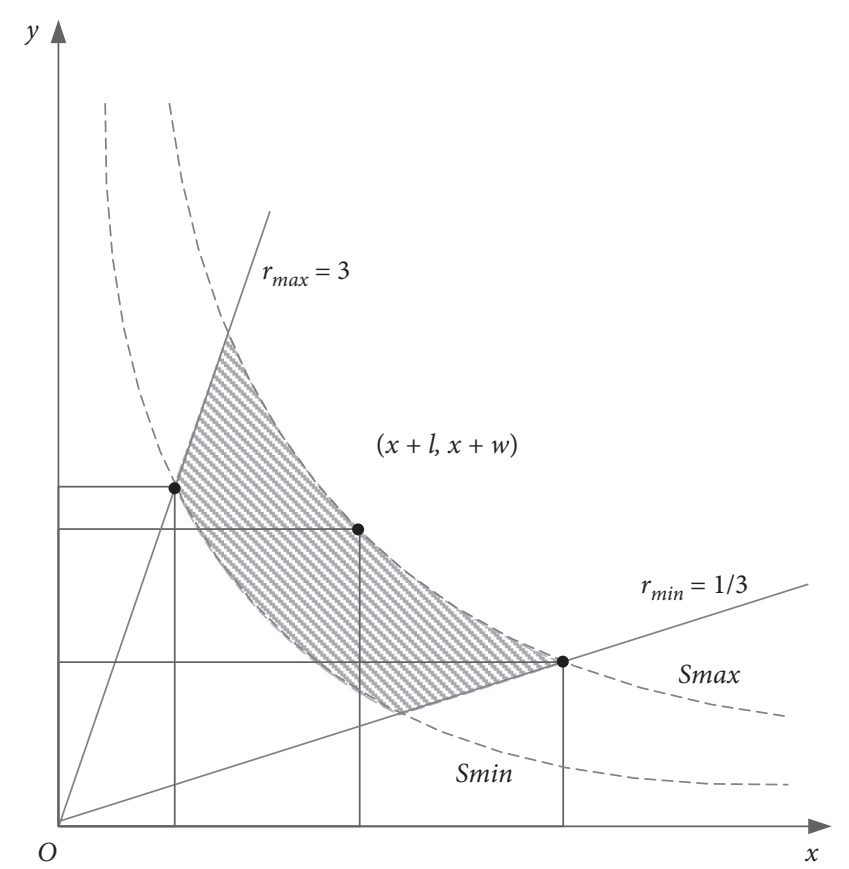

FIgURE 6: The difference caused by the dimension of arranged objects.

The difference in ship deck arrangements caused by the positional variables may be more pronounced. Different positions of arranged objects may create different relativelocation relationships, which may result in completely different separation and adjacency satisfactions, and most of the position variables can vary throughout the deck space.

For measuring the arrangement similarity of the same arranged object in two different ship deck arrangements, a new concept, topological similar zone, is proposed, as shown in Figure 7. The reference point $\left(x_{k}^{z}, y_{k}^{z}\right)$ of the topological similar zone of the arranged object $E_{k}$ is determined by equation (30), where the input parameter $\lambda_{t}$ is used to determine the size of this topological similar zone. The length $l_{i}^{z}$ and width $w_{i}^{z}$ of the topological similar zone can be calculated according to equation (31). For the same arranged object $E_{k}$ in two different ship deck arrangements, if they are both within the topological similar zone of each other, the element $E_{k}$ can be arranged at a similar position in the two arrangements. And the arrangement similarity $\varphi_{k}$ of the arranged object $E_{k}$ in these two different arrangements would be equal to 1 ; otherwise, $\varphi_{k}$ would be equal to 0 . In consequence, the diversity $D_{v_{-} i j}$ of two ship deck arrangements (individuals) $i$ and $j$ could be calculated based on the arrangement similarity of arranged objects, which is expressed by equation (32). And the minimum value of diversity $D_{v_{-} i j}$ may be used to represent the diversity $D_{v}(i)$ of the individual $i$ in the population, as shown in equation (32). If the individual $i$ ranks first in the population, the diversity of the individual $i$ would be equal to 1 . After the diversity of all individuals in the population is calculated, a replacement threshold $\lambda_{r}$ is determined by designers to eliminate similar individuals. The individuals with diversity less than the replacement threshold $\lambda_{r}$ are eliminated and 


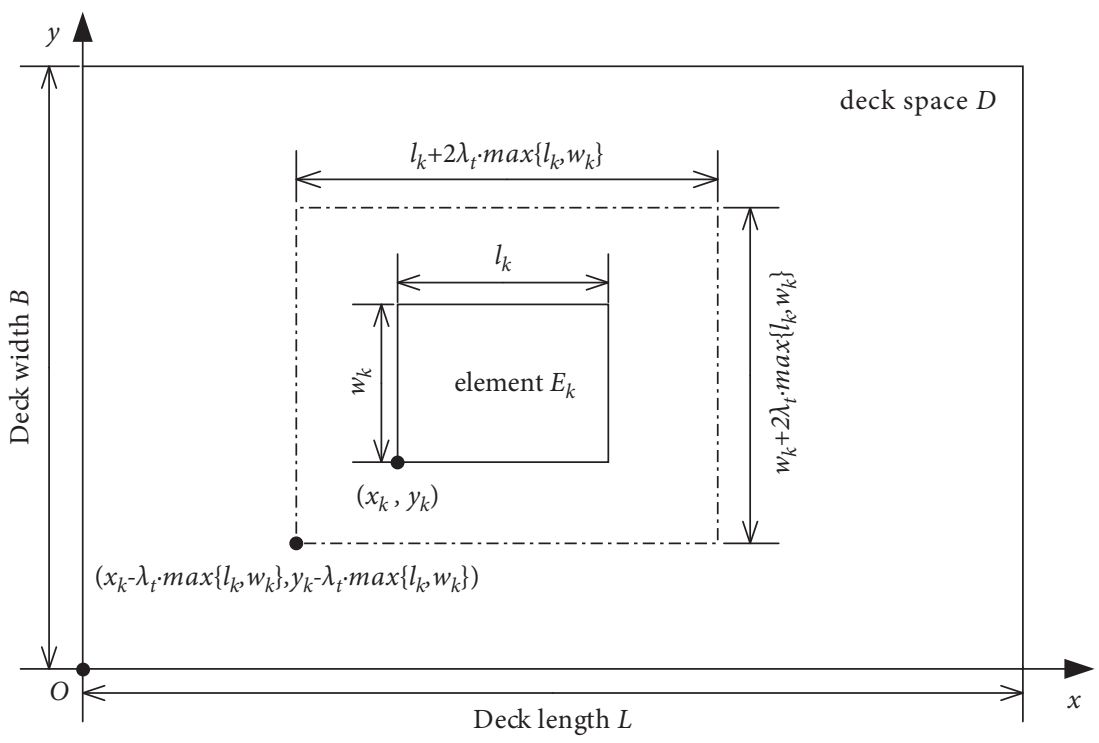

FIgURE 7: Topological similar zone.

new individuals randomly generated are added to the population to maintain the size of the population.

$$
\begin{gathered}
\left\{\begin{array}{l}
x_{k}^{z}=x_{k}-\lambda_{t} \cdot \max \left\{l_{k}, w_{k}\right\}, \\
y_{k}^{z}=y_{k}-\lambda_{t} \cdot \max \left\{l_{k}, w_{k}\right\},
\end{array}\right. \\
\left\{\begin{array}{l}
l_{k}^{z}=l_{k}+2 \times \lambda_{t} \cdot \max \left\{l_{k}, w_{k}\right\}, \\
w_{k}^{z}=w_{k}+2 \times \lambda_{t} \cdot \max \left\{l_{k}, w_{k}\right\},
\end{array}\right. \\
D_{v_{-} i j}=1-\sum_{k=1}^{N} \frac{\varphi_{k}}{n}, \\
D_{v}(i)=\min \left(D_{v_{-i j}}\right), \quad j=1,2, \ldots,(i-1) .
\end{gathered}
$$

\section{Computational Results}

To verify the validity of the improved algorithm to solve the ship deck arrangement optimization problem, the proposed algorithm and the standard NSGA-II were used to solve the same instance for comparison. Then, the performance of the proposed algorithm and the behaviors of the adaptive crossover and topological replace operators were tested. All tests were implemented in MATLAB 2014b and run on a Windows 10 Pro computer with Intel ${ }^{\circledR}$ Core $^{\mathrm{TM}}$ i7-6700K CPU @4.00 GHz.

4.1. Description of the Instance. The main deck arrangement of an underwater detection ship is chosen as an instance to test the validity of the proposed algorithm in ship deck arrangement optimization. The underwater detection ship is 47-meter long and 12.2-meter wide, and 27 arranged objects would be arranged on its main deck. The arranged objects to be included in the main deck arrangement optimization are presented in Table 3.
Although the feasible domains of the positional variables can be determined directly by the deck space, designers may define more strict feasible domains of positional variables to make the ship deck arrangements closer in practice. The specific feasible domains of positional variables of arranged objects are listed in Table 3. The diving platform is special equipment used by divers to dive, so it must be arranged at the ship stern end. The slewing anchors, No. 1 and No. 2 position anchors, must be arranged at the ship bow end. And the No. 1 and No. 2 position anchors should be symmetrically arranged for stable positioning. A linear equality constraint expressed by equation (34) is used to ensure that the two arranged objects $E_{i}$ and $E_{j}$ are symmetrically arranged. The No. 3 and No. 4 position anchors do not have a fixed longitudinal position but should be arranged close to the ship stern end. And they must be symmetrically arranged at the edge of the deck. Similarly, the No. 1 and No. 2 main corridors are fixedly arranged at the edge of the deck in the design. Except for anchors and diving platform, the arranged objects cannot be arranged at the working zone of the cranes for safety reasons. As No. 2 crane is arranged in front of the No. 1 crane, the working zone of the No. 1 crane is close to the ship stern end and its working radius is 12 meters, and the working zone of the No. 2 crane is close to the ship bow end and its working radius is 8 meters. The special arrangement design requirements for the cranes $E_{20}$ and $E_{21}$ are expressed by equations (35) and (36). It can be observed that there are 19 arranged objects with completely uncertain positions and 2 arranged objects with fully fixed positions and the remaining 6 arranged objects have fixed $x$ or $y$ variables. A total of 44 uncertain positional variables are involved in the optimization.

$$
\begin{aligned}
y_{i}+y_{j}-12.2 & =0, \\
x_{i}-x_{20}<0, & i \in\{1,2, \ldots, 19\} \\
x_{i}+l_{i}-x_{21}-l_{21}<0, & i \in\{1,2, \ldots, 19\} .
\end{aligned}
$$


Table 3: Arranged objects to be included in the optimization.

\begin{tabular}{|c|c|c|c|c|}
\hline List of arranged objects & {$\left[x_{\text {low }}, x_{\text {up }}\right]$} & {$\left[y_{\text {low }}, y_{\text {up }}\right]$} & {$\left[s_{\text {low }}, s_{\text {up }}\right]$} & {$\left[r_{\text {low }}, r_{\text {up }}\right]$} \\
\hline Detection equipment cabin & {$[0,40]$} & {$[0,12.2]$} & {$[10.4,17.4]$} & {$[0.5,2]$} \\
\hline No. 1 working cabin & {$[0,40]$} & {$[0,12.2]$} & {$[10,20]$} & {$[0.5,2]$} \\
\hline No. 2 working cabin & {$[0,40]$} & {$[0,12.2]$} & {$[10,20]$} & {$[0.5,2]$} \\
\hline Galley & {$[0,40]$} & {$[0,12.2]$} & {$[8.3,13.9]$} & {$[0.5,2]$} \\
\hline Mess & {$[0,40]$} & {$[0,12.2]$} & {$[13.5,22.5]$} & {$[0.5,2]$} \\
\hline Storeroom & {$[0,40]$} & {$[0,12.2]$} & {$[3,6]$} & {$[0.5,2]$} \\
\hline Emergency generator room & {$[0,40]$} & {$[0,12.2]$} & {$[9.8,16.3]$} & {$[0.5,2]$} \\
\hline Engine case & {$[0,40]$} & {$[0,12.2]$} & {$[13.5,22.5]$} & {$[0.5,2]$} \\
\hline Changing room & {$[0,40]$} & {$[0,12.2]$} & {$[13.5,22.5]$} & {$[0.5,2]$} \\
\hline Diving equipment cabin & {$[0,40]$} & {$[0,12.2]$} & {$[6.5,10.9]$} & {$[0.5,2]$} \\
\hline Toilet & {$[0,40]$} & {$[0,12.2]$} & {$[5,8]$} & {$[0.5,2]$} \\
\hline Internal corridor & {$[0,40]$} & {$[0,12.2]$} & {$[5,8]$} & {$[0.3,3]$} \\
\hline No. 1 detecting sonar & {$[0,40]$} & {$[1,11.2]$} & $\{2.2\}$ & $\{2.2\}$ \\
\hline No. 2 detecting sonar & {$[0,40]$} & {$[1,11.2]$} & $\{2.2\}$ & $\{2.2\}$ \\
\hline Submersible decompression cabin & {$[0,40]$} & {$[2.4,9.8]$} & $\{15.12\}$ & $\{2.63\}$ \\
\hline No. 1 stair & {$[0,40]$} & {$[0,12.2]$} & $\{2.56\}$ & $\{0.25,4\}$ \\
\hline No. 2 stair & {$[0,40]$} & {$[0,12.2]$} & $\{2.56\}$ & $\{0.25,4\}$ \\
\hline No. 1 main corridor & {$[8.6,38]$} & $\{0\}$ & $\{36\}$ & $\{16\}$ \\
\hline No. 2 main corridor & {$[8.6,38]$} & $\{10.7\}$ & $\{36\}$ & $\{16\}$ \\
\hline No. 1 crane & {$[8.6,24]$} & {$[1.7,10.5]$} & $\{5.1\}$ & $\{1.77\}$ \\
\hline No. 2 crane & {$[24,40]$} & {$[1.7,10.5]$} & $\{5.1\}$ & $\{1.77\}$ \\
\hline No. 1 position anchor & $\{40\}$ & {$[6.1,12.2]$} & $\{17.5\}$ & $\{2.8\}$ \\
\hline No. 2 position anchor & $\{40\}$ & {$[0,6.1]$} & $\{17.5\}$ & $\{2.8\}$ \\
\hline No. 3 position anchor & {$[2.6,8.6]$} & $\{0\}$ & $\{17.5\}$ & $\{2.8\}$ \\
\hline No. 4 position anchor & {$[2.6,8.6]$} & $\{9.7\}$ & $\{17.5\}$ & $\{2.8\}$ \\
\hline Slewing anchor & $\{41\}$ & $\{5.1\}$ & $\{12\}$ & $\{3\}$ \\
\hline Diving platform & $\{0\}$ & $\{4.8\}$ & $\{16.9\}$ & $\{2.5\}$ \\
\hline
\end{tabular}

According to the function, arranged objects can be divided into two categories: cabin and deck machinery. Generally, the dimensions of deck machinery are fixed, meaning that their length, width, area, and aspect ratio are constant in the optimization. And the dimensions of the cabins are uncertain and their length and width are depended on their area and aspect ratio. Therefore, ship designers need to determine the reasonable area and aspect ratio ranges of cabins. The reasonable area range of cabins could be decided according to ship design standards, experience, and shipowner's requirements. The aspect ratio range of cabins is related to their internal layout. However, in the early stage of ship design, it is hard to obtain the details of the cabin layout. Finally, the aspect ratio of all cabins on the underwater detection ship is limited to between 0.5 and 2 to avoid generating unreasonable cabins that are too narrow. It can be observed that there are 12 arranged objects with completely uncertain dimensions and the remaining 15 arranged objects have fully fixed dimensions. Since the stair with fixed dimensions may vary in orientation, the aspect ratio of the stair would be selected from the two values of 0.25 and 4 to distinguish its orientation. Figure 8 shows that the stair with an aspect ratio of 0.25 is transversely arranged in the deck space, and the stair with an aspect ratio of 4 is longitudinally arranged in the deck space. The specific feasible domains of the area and aspect ratio of the arranged objects are listed in Table 3 . The main deck arrangement optimization would involve 28 uncertain dimensional variables.

Finally, this instance involves 72 uncertain design variables, including 44 positional variables and 28-dimensional variables. And a total of 27 arranged objects are included in the main deck arrangement, so theoretically 351 nonlinear equality constraints (overlapping constraints) must be used in the optimization. However, some of the arranged objects have limited arrangement zones and do not overlap each other. In consequence, a total of 222 nonlinear equality constraints are actually used in this instance.

4.2. Computational Results of the Validation Tests. For comparing the validity, the IMOHGA and standard NSGAII are used to solve the main deck arrangement optimization problem described above. In the validation tests, the two algorithms run on the same input data. Table 2 lists parts of the input data, including mathematical model parameters and algorithm parameters; Table 3 lists the specific feasible domains of design variables; and equations (37)-(39) present the material flow frequency matrix Mf, material flow cost matrix $\mathrm{Mc}$, and separation relationship matrix $\mathrm{Mr}$, respectively. The iteration convergence behaviors of the two algorithms are registered. Figures 9(a) and 9(b) illustrate the convergence features of the nonlinear (overlapping) constraint violations. It can be observed that the standard NSGA-II could not generate feasible individuals if all overlapping constraints are met. However, the IMOHGA searched feasible individuals after nearly 100 iterations. The convergence features of the IMOHGA in searching the minimum optimization objectives may be observed in Figure 10, where the best topological and geometric 


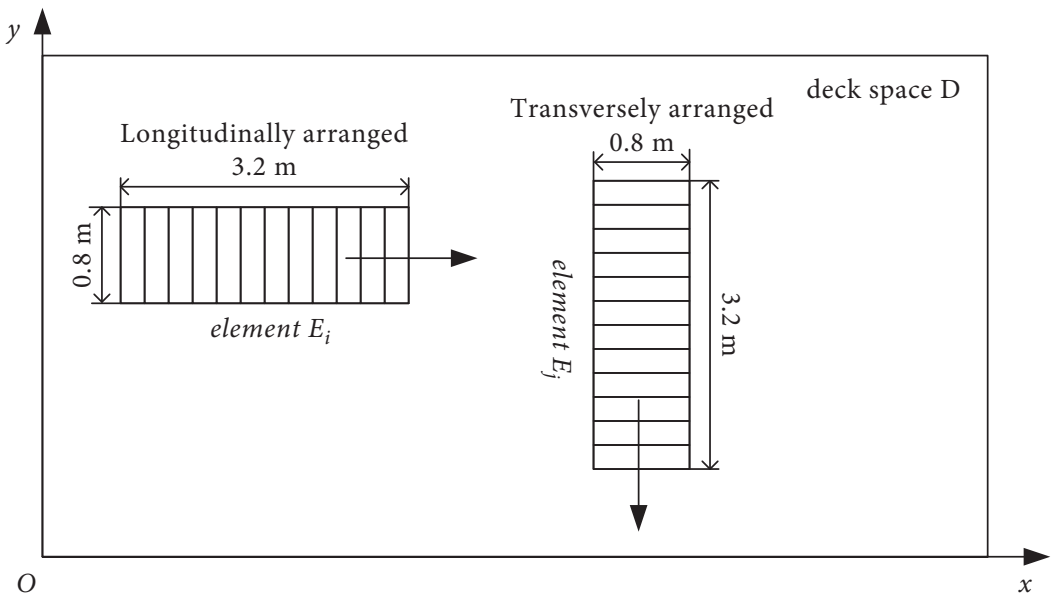

FIgURe 8: Two possible configurations of the ship stairs.
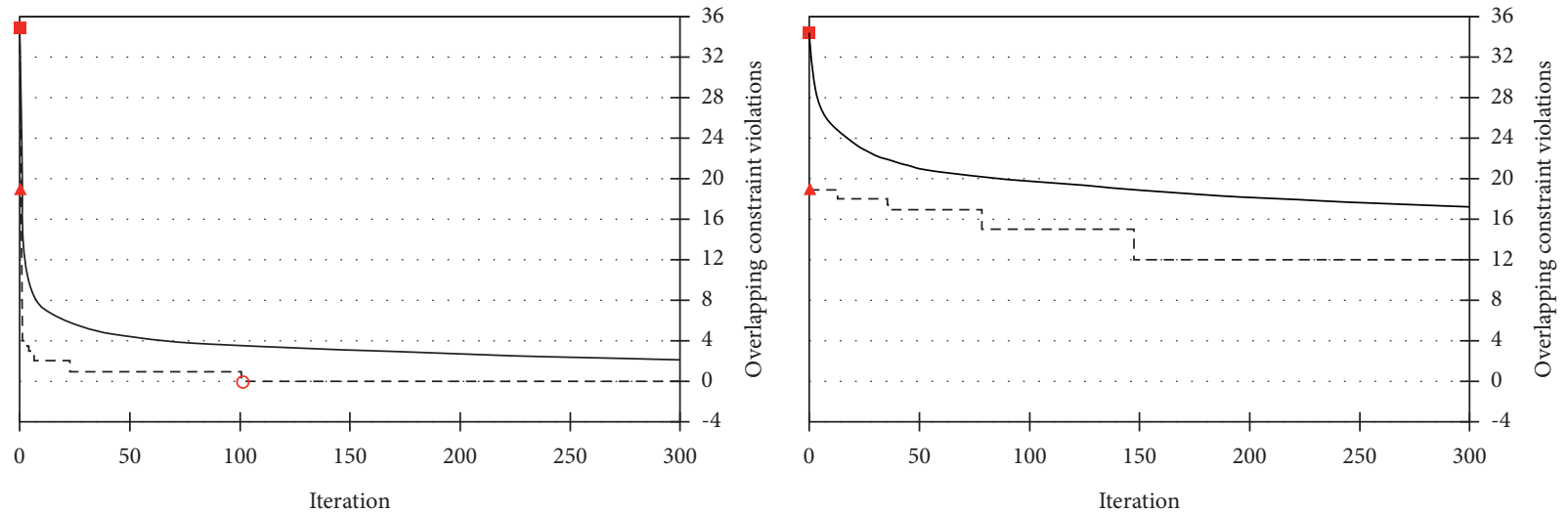

The average number of violations

The average number of violations

- The average number of violations in the initial population

- The average number of violations in the initial population

- - - The minimum number of violations

- - - The minimum number of violations

$\Delta$ The minimum number of violations in the initial population

(a)

A The minimum number of violations in the initial population

(b)

FIGURE 9: Iteration convergence feature of the nonlinear constraint violations. (a) Iteration of the IMOHGA. (b) Iteration of the standard NSGA-II.

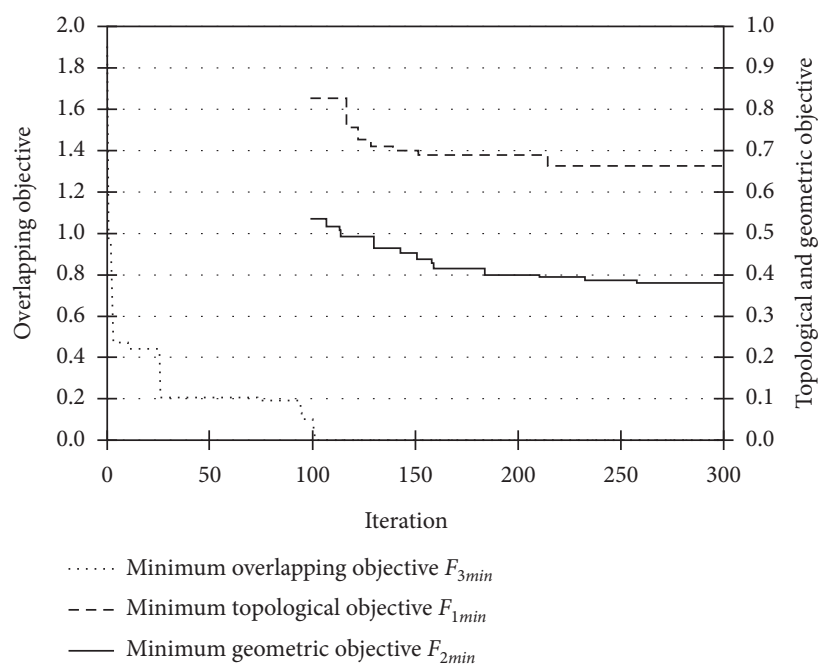

FIGURE 10: Iteration convergence feature of the IMOHGA in searching the minimum objectives. 
objectives $\left(F_{1 \min }\right.$ and $\left.F_{2 \min }\right)$ are for the feasible individuals. The three-dimensional main deck arrangements corresponding to the six optimal solutions in the Pareto-optimal group are displayed in Figure 11. For ease of identification, the cranes are represented by two spliced cuboids, while other arranged objects are represented by a single cuboid.

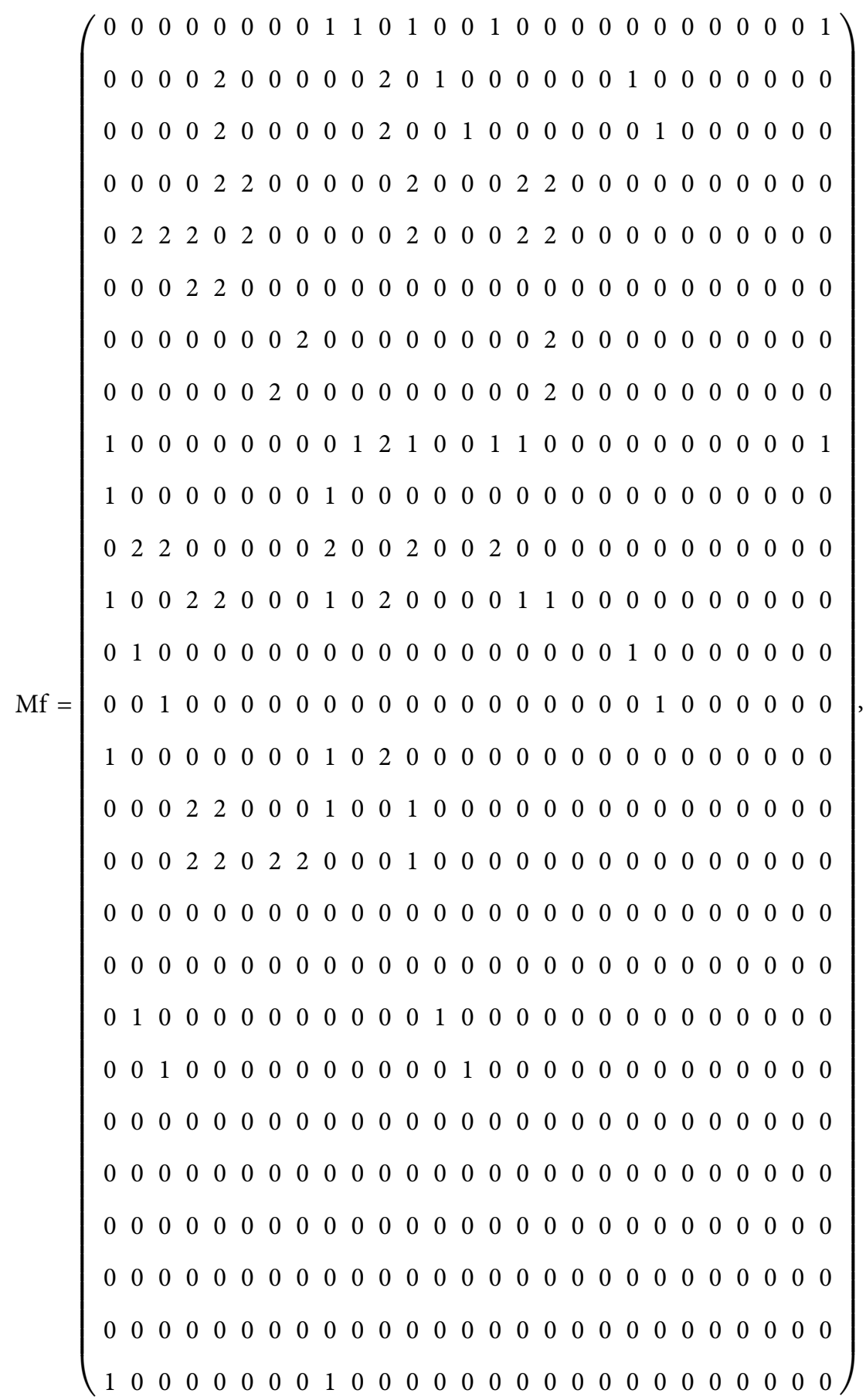



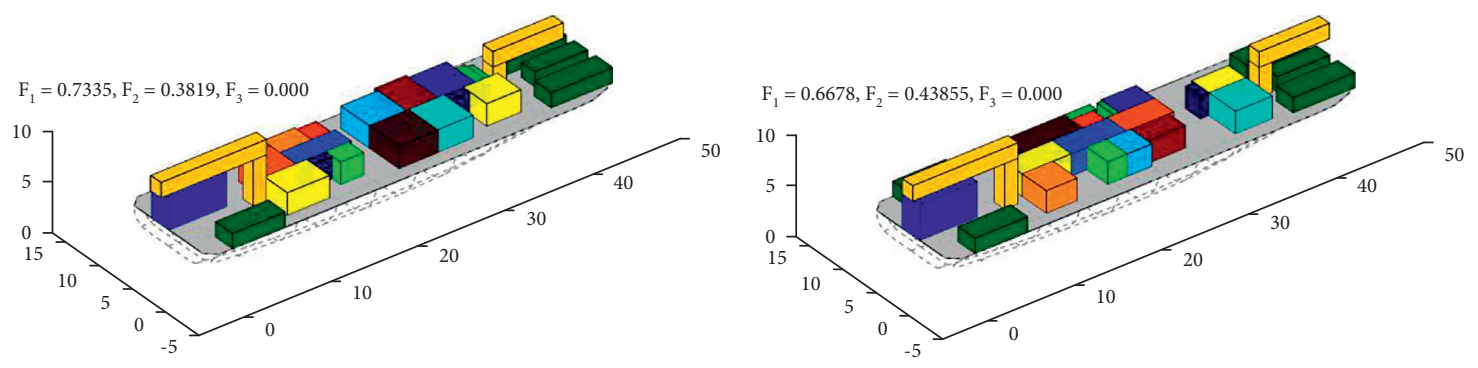

$$
\begin{array}{ll}
\square \text { Toilet x 1 } & \text { Emergency } \\
\square \text { Stair x 2 } & \text { Drtecing s } \\
\square \text { Anchor x 5 } & \text { Engine Ca } \\
\square \text { Working cabin x 2 } & \square \text { Changing } \\
\square \text { Crane x 2 } & \square \text { Diving equ } \\
\square \text { Galley x 1 } & \square \text { Submersib } \\
\square \text { Mess x 1 } & \text { Diving plat } \\
\square \text { Storeroom x 1 } & \text { Detection } \\
\square \text { Corridor x 3 (The corridors } \\
\text { would not display in the arrangements) }
\end{array}
$$

(a)

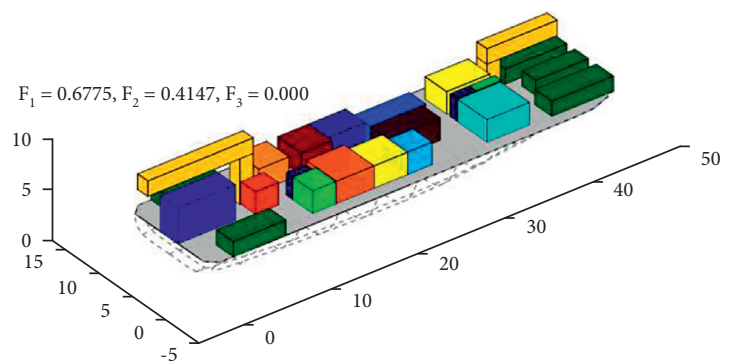

$$
\begin{array}{ll}
\square \text { Toilet x 1 } & \square \text { Emergency generator room x 1 } \\
\square \text { Stair x 2 } & \text { Drtecing sonar x 2 } \\
\square \text { Anchor x 5 } & \text { Engine Case x 1 } \\
\square \text { Working cabin x 2 } & \square \text { Changing room x 1 } \\
\square \text { Crane x 2 } & \square \text { Diving equipment cabin x 1 } \\
\square \text { Galley x 1 } & \square \text { Submersible decompression chamber x 1 } \\
\square \text { Mess x 1 } & \square \text { Diving platform x 1 } \\
\square \text { Storeroom x 1 } & \square \text { Detection equipment cabin x 1 } \\
\square \text { Corridor x 3 (The corridors } \\
\text { would not display in the arrangements) }
\end{array}
$$

(c)

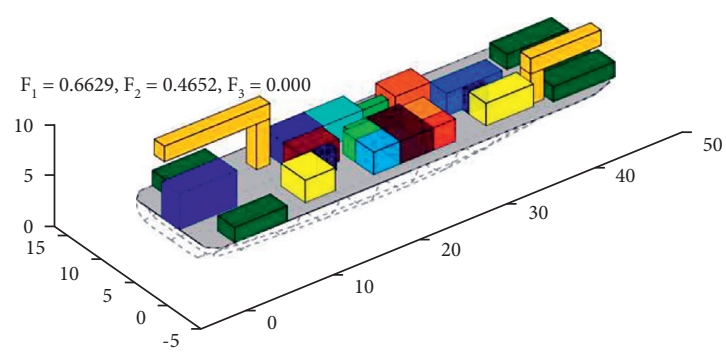

$$
\begin{array}{ll}
\square \text { Toilet x 1 } & \square \text { Emergency generator room x 1 } \\
\square \text { Stair x 2 } & \text { Drtecing sonar x 2 } \\
\square \text { Anchor x 5 } & \text { Engine Case x 1 } \\
\square \text { Working cabin x 2 } & \square \text { Changing room x 1 } \\
\square \text { Crane x 2 } & \square \text { Diving equipment cabin x 1 } \\
\square \text { Galley x 1 } & \square \text { Submersible decompression chamber x 1 } \\
\square \text { Mess x 1 } & \square \text { Diving platform x 1 } \\
\square \text { Storeroom x 1 } & \text { Detection equipment cabin x 1 } \\
\square \text { Corridor x 3 (The corridors } \\
\text { would not display in the arrangements) }
\end{array}
$$

(e)

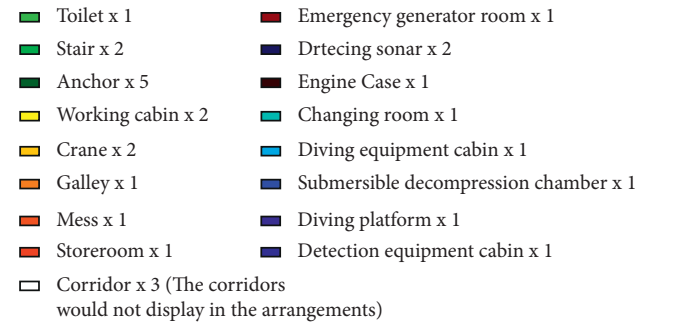

(b)

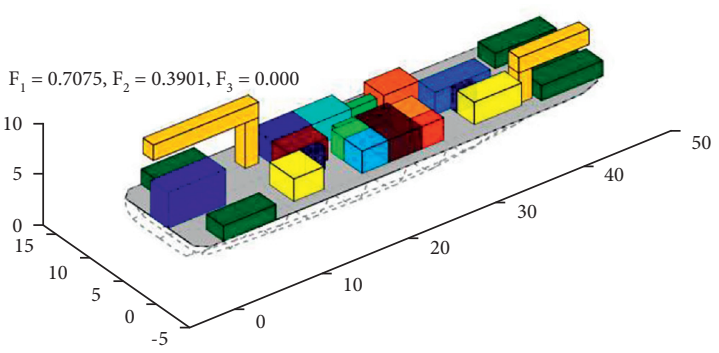

$$
\begin{array}{ll}
\square \text { Toilet x 1 } & \text { Emergency generator room x 1 } \\
\square \text { Stair x 2 } & \text { Drtecing sonar x 2 } \\
\square \text { Anchor x 5 } & \text { Engine Case x 1 } \\
\square \text { Working cabin x 2 } & \text { Changing room x 1 } \\
\square \text { Crane x 2 } & \square \text { Diving equipment cabin x 1 } \\
\square \text { Galley x 1 } & \text { Submersible decompression chan } \\
\square \text { Mess x 1 } & \text { Diving platform x 1 } \\
\square \text { Storeroom x 1 } & \text { Detection equipment cabin x 1 } \\
\square \text { Corridor x 3 (The corridors } \\
\text { would not display in the arrangements) }
\end{array}
$$

(d)

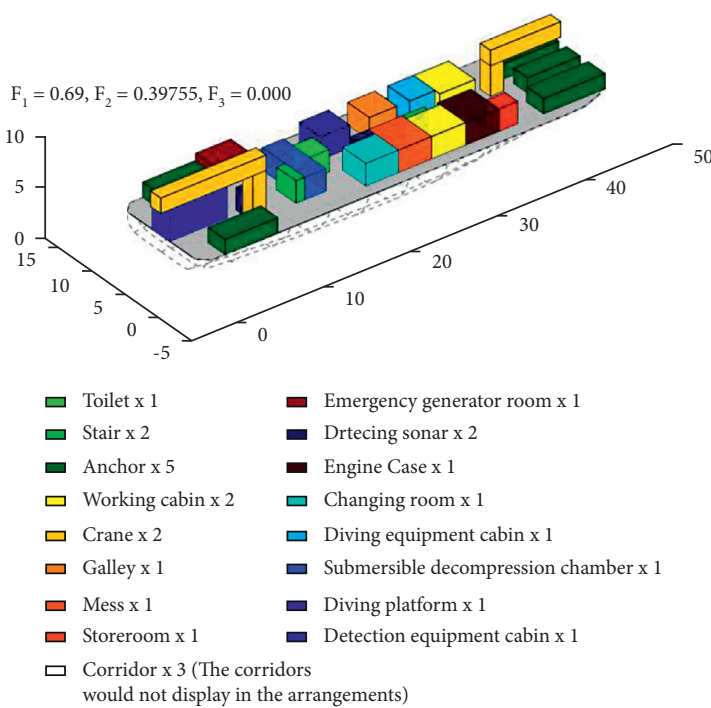

(f)

FIGURE 11: Six main deck arrangements of the underwater detection ship in the Pareto-optimal group. 


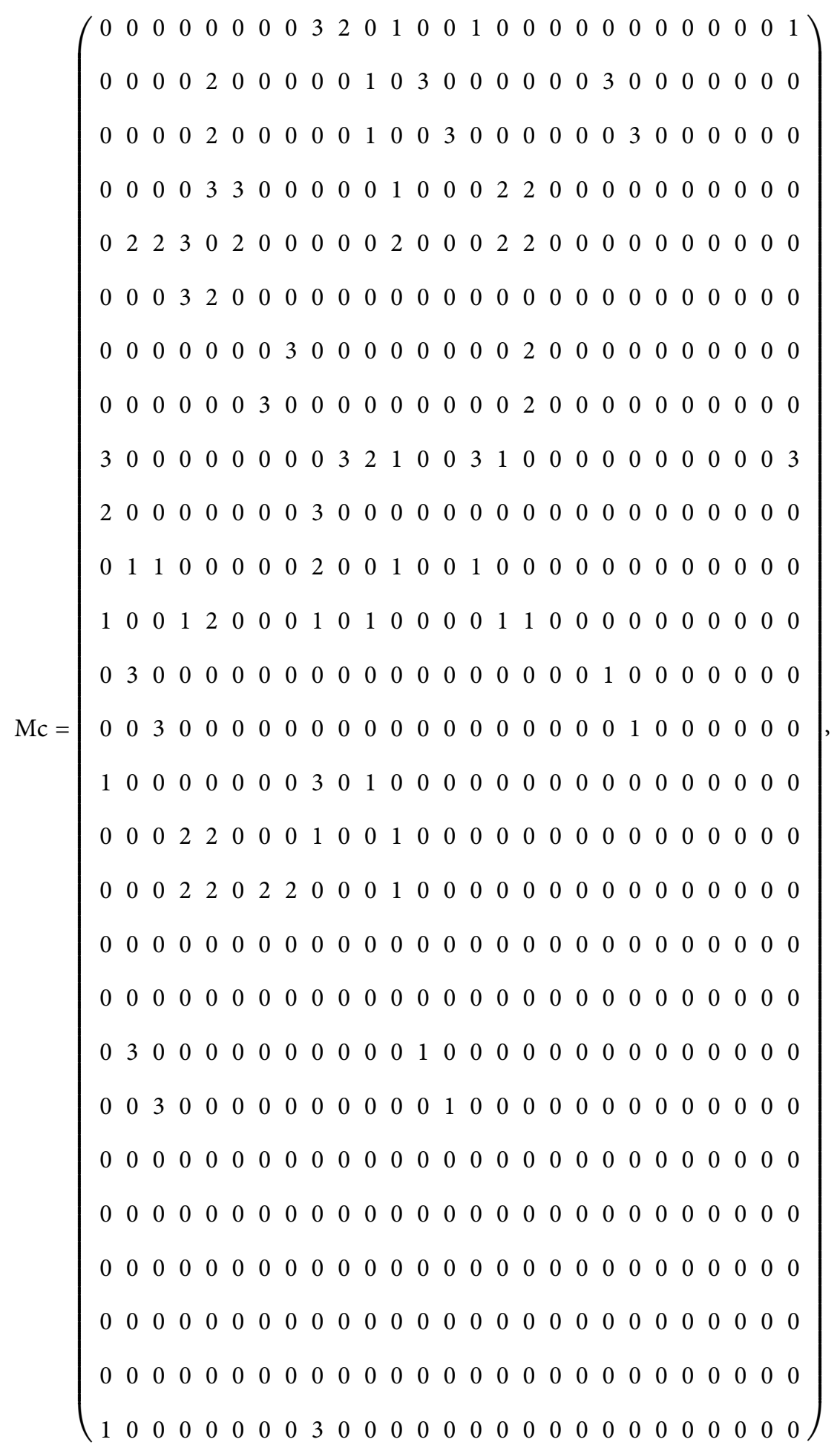




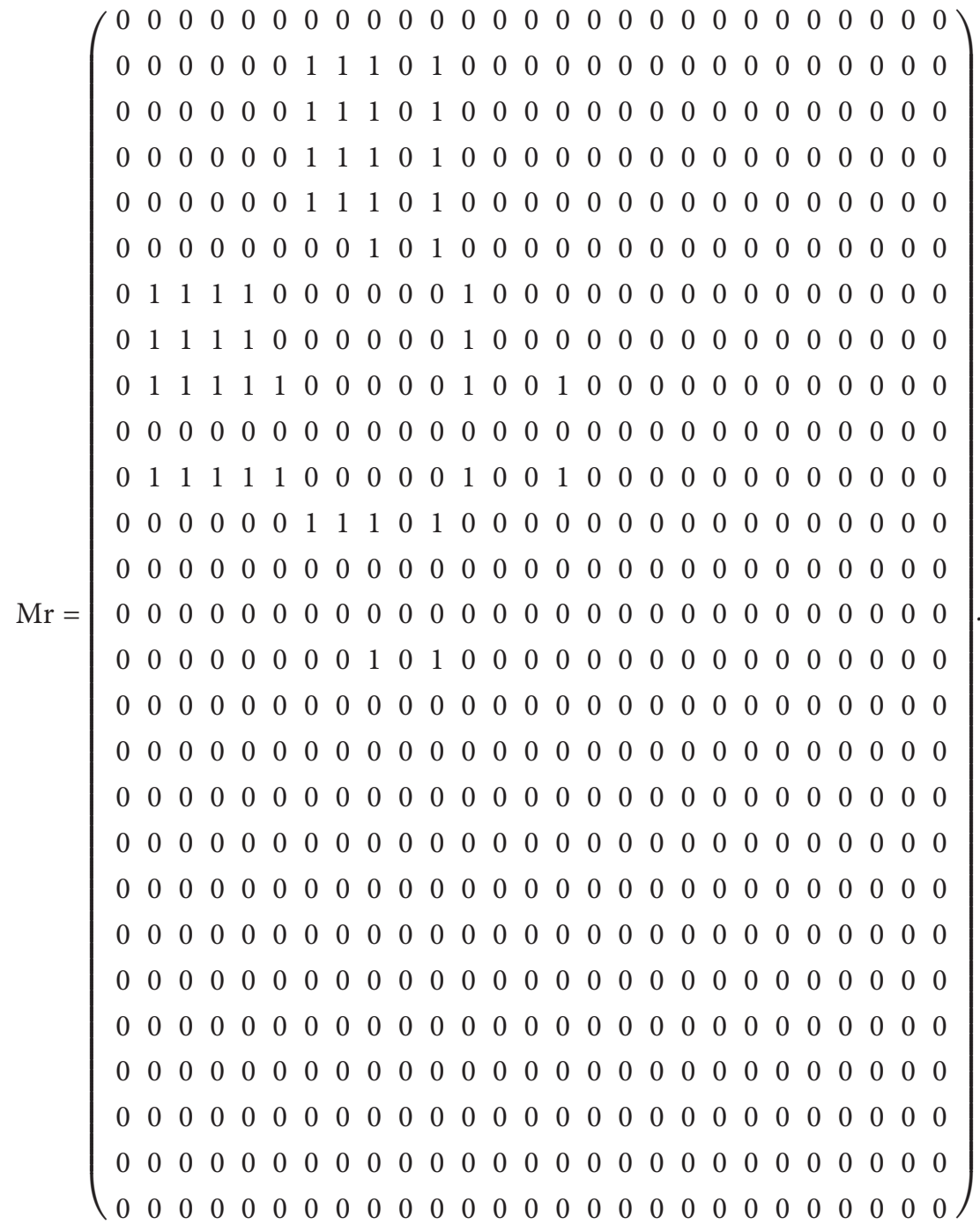

4.3. Computational Results of the Performance Tests. With the purpose of determining the nonlinear constraint handling performance of the proposed algorithm and its robustness, the proposed algorithm was run 10 times for the main deck arrangement optimization problem using the input data in the validation tests. In the performance tests, the behaviors of the population and the feasible individual group throughout the search process and the running time of the algorithm were registered. The performance indicators used to assess the capability of nonlinear constraint handling were the average number $\left(c_{\text {avg }}\right)$ of nonlinear constraint violations in the population and the minimum number $\left(c_{\min }\right)$ of nonlinear constraint violations in the population. And the averages $\left(\bar{c}_{\text {avg }}\right.$ and $\left.\bar{c}_{\text {min }}\right)$ of these two performance indicators from those 10 runs were calculated and illustrated in Figure 12. In this figure, the green circle marks the minimum iterations to find the feasible individual in those 10 runs and the red circle marks the maximum iterations. In addition, the average $\left(\bar{F}_{1 \mathrm{~min}}\right.$ and $\left.\bar{F}_{2 \text { min }}\right)$ of the minimum topological and geometric objective values of the feasible individuals' group from those 10 runs were also calculated to assess the algorithm. Of course, the overlapping objective of the feasible individuals must be zero. Figure 13 presents a box plot of the four performance indicators $\left(\bar{c}_{\text {avg }}, \bar{c}_{\text {min }}, \bar{F}_{1 \text { min }}\right.$, and $\left.\bar{F}_{2 \text { min }}\right)$ to assess the robustness of the algorithm. The diversity of the population and Pareto-optimal group could be observed in Figures 14 and 15. And the average running time of the hybrid genetic algorithm for this problem was 4168.3 seconds and the maximal and minimal running times of that were 4324.6 seconds and 3991.2 seconds.

Subsequently, to verify the effect of the adaptive crossover operator, the IMOHGA using the multiple-point crossover operator would be run 10 times and the IMOHGA using the simulated binary crossover operator would be also run 10 times for comparison. The averages of performance indicators $c_{\text {avg }}$ and $c_{\text {min }}$ from the two sets of 10 runs were calculated and illustrated in Figures 16(a) and 16(b). In the figures, the green circle marks the minimum iteration to find the feasible individuals in the 10 runs and the red circle marks the maximum iteration. And the average diversity $\left(D_{v_{-} \text {ave }}\right.$ and $D_{v_{-} \text {opt }}$ ) of the population and the average diversity of the Pareto-optimal group were two indicators to measure the ability of the different crossover operators to maintain the diversity of the population. The averages 


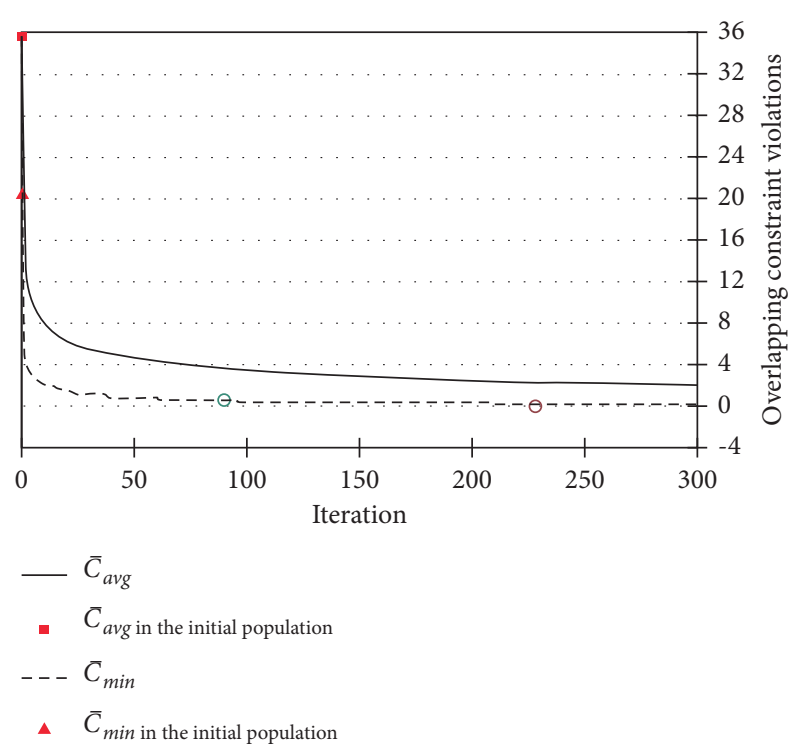

FIGURE 12: The performance of the IMOHGA in handling overlapping constraints.

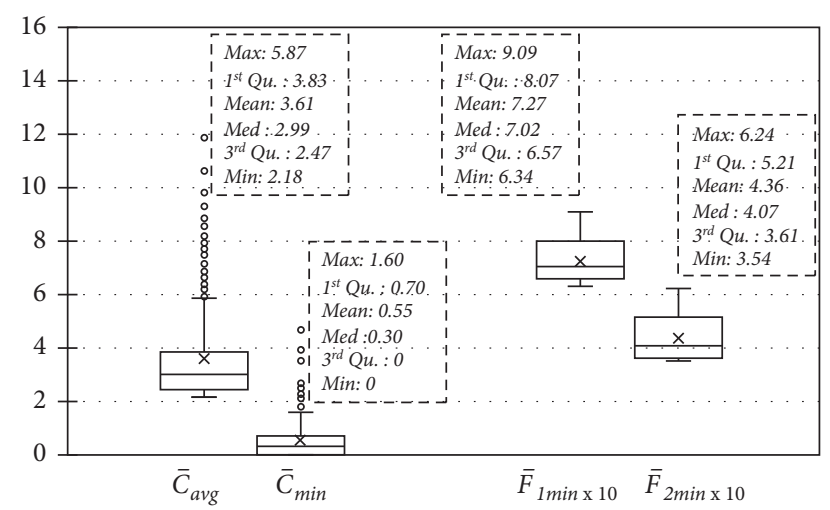

FIGURE 13: Box plot of performance indicators, $\bar{c}_{\text {avg }}, \bar{c}_{\text {min }}, \bar{F}_{1 \text { min }}$, and $\bar{F}_{2 \min }$.

$\left(\bar{D}_{v_{-} \text {ave }}\right.$ and $\left.\bar{D}_{v_{-} \text {opt }}\right)$ of population and Pareto-optimal group diversity indicators from the three sets of 10 runs were calculated and shown in Figures 14(a) and 14(b).

Finally, the validity of the topological replace operator is tested. The proposed algorithm would be run another 10 times without the topological replace operator for comparison. The averages $\left(\bar{D}_{v_{-} \text {ave }}\right.$ and $\left.\bar{D}_{v_{-} \text {opt }}\right)$ of population and Pareto-optimal group diversity indicators from these 10 runs were calculated and compared in Figures 15(a) and 15(b).

\section{Discussions}

5.1. The Deck Arrangements of the Underwater Detection Ship. As shown in Figure 11, the diverse arrangement results are obviously difficult to directly apply to the general arrangement design of the ship. It is a bit subjective to define what a good arrangement is in this problem. To measure how well the generated arrangements are, three objective functions are defined. It can be observed that, after optimization, the topological objective function can be reduced from 0.82 to 0.66 , and the geometric objective function can be reduced from 0.54 to 0.38 . Table 4 summarizes the remarks of the six arrangements for four aspects by ship designers. The first aspect is the relationship between galley and mess. Generally, the mess should be close to the galley. The second aspect is the relationship between the toilet and the galley and mess. The toilet is required to be away from the galley and mess to improve the dining environment. The next aspect is the relationship between the working cabins and the cranes. The working cabins need to have a good view for the operation of the cranes. And the last aspect is the layout of the diving system. For this underwater detection ship, the diving system consists of a detection equipment cabin, diving platform, and submersible decompression cabin. Therefore, these cabins should be arranged relatively adjacent to facilitate diving work.

The remarks listed in Table 4 indicate that the deck arrangements generated by the proposed algorithm are not perfect. The main reason is that there are a lot of simplifications in the topological and geometric objectives. Due to the spiraling characteristics of the ship design process, designers can select a suitable arrangement from the optimization results for the detailed design. The final arrangement of the underwater detection ship used in this paper is developed from Figure 11(d), and the detailed deck arrangement plan of the real ship and the deck arrangement plan of Figure 11(d) are compared in Figure 17.

It can be observed from the real ship's arrangements that it can obtain a competitive general arrangement based on the optimization results generated by the proposed algorithm. However, there is some difference between the deck arrangements in the different stages of ship design. The first major difference is the minor adjustment of the dimensions and positions of the arranged objects. This adjustment aims to make the deck arrangement tidier and adapt to the structure of the ship. And the second major difference is the cabins with irregular shapes on the deck, such as the working cabin and emergency generator room. The irregular shape of the working cabin (aft) ensures that the corridor is unobstructed; the irregular shape of the emergency generator room and the working cabin (fore) is to avoid interference with the adjusted detecting sonar. In addition, the windows and doors of ship cabins are not considered in the optimization.

In the early stage of ship design, there is very little information that could be used for determining the deck arrangement, so it makes sense to develop an approach to automatically generate the deck arrangements, especially for the special and new ship types. There is almost no parent ship information. It is a time-consuming task to generate $s$ set of competitive general arrangements by using the traditional design methods in the early stage of ship design.

\subsection{The Initial Population Size and Maximum Iterations.} The main purpose of the validation and performance tests is to determine the capability of the IMOHGA to generate diverse and rational deck arrangements. Due to the fact that 


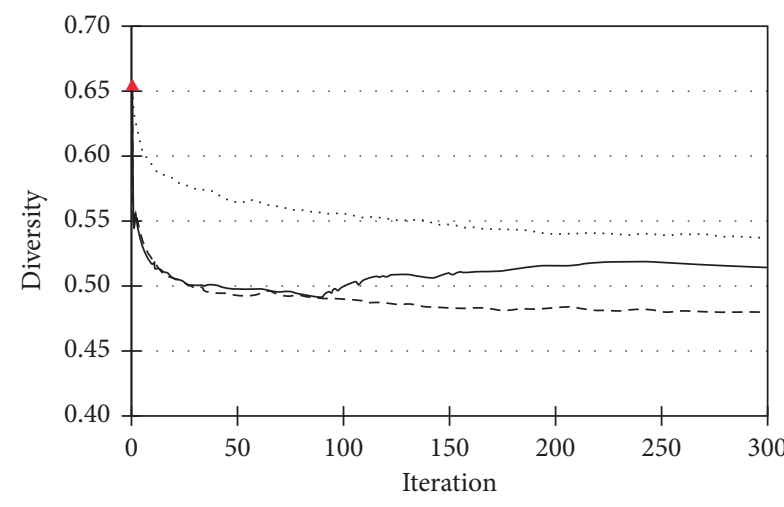

The average diversity of population
(Adaptive crossover)
$\ldots$. The average diversity of population
Multiple-point crossover)
--- The average diversity of population
(Simulated binary crossover)

(a)

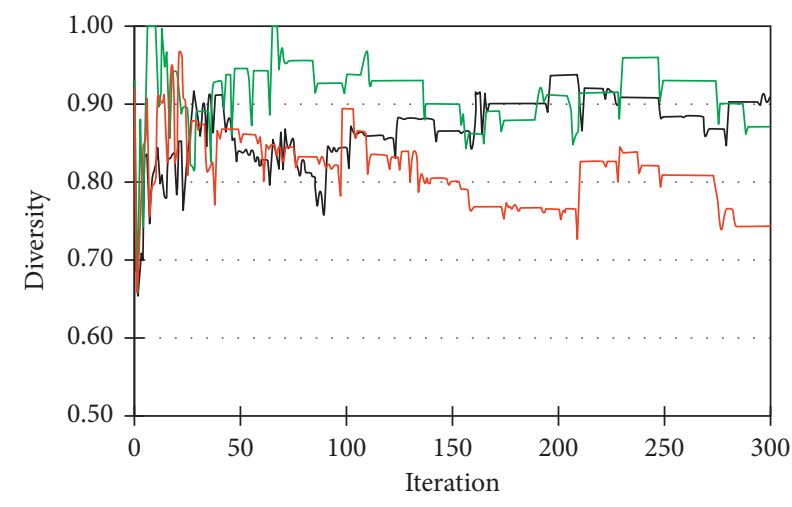

The average diversity of Pareto-optimal group
(Adaptive crossover)
The average diversity of Pareto-optimal group
(Multiple-point crossover)
The average diversity of Pareto-optimal group
(Multiple-point crossover)

(b)

Figure 14: The influence of different crossover operators on performance indicators, $\bar{c}_{\text {avg }}$ and $\bar{c}_{\min }$. (a) The average diversity of the population. (b) The average diversity of the Pareto-optimal group.

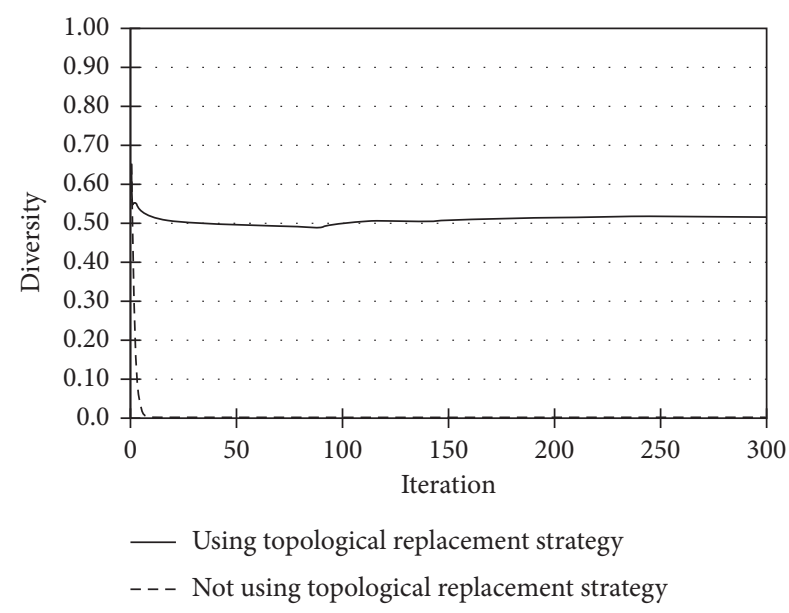

(a)

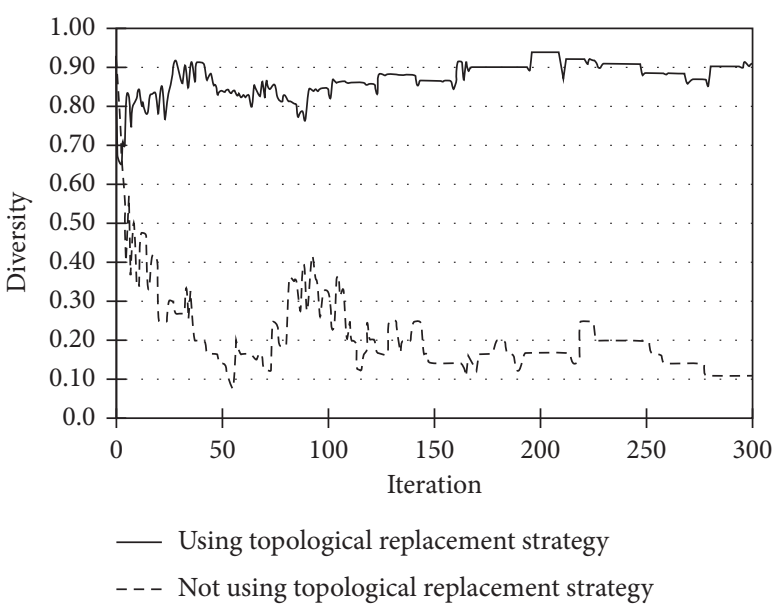

(b)

FIGURE 15: Influence of topological replace operator on the population and Pareto-optimal group diversity. (a) The average diversity of the population. (b) The average diversity of the Pareto-optimal group.

the arrangement optimization of the specific instance is a highly constrained optimization problem, which includes 72 uncertain optimization variables and 222 nonlinear constraints, it is necessary to predefine the sufficiently large initial population size and maximum iterations for consistent comparison.

The value is 200 for the initial population size to reduce the influence of the randomness of the initial population. Figure 18 illustrates the minimum number $c_{0 \text { min }}$ and the average number $c_{0 \text { avg }}$ of nonlinear constraint violations in the initial population generated in the four sets of performance tests. The average values $\bar{c}_{0 \text { min }}$ in these four sets of performance tests are 20.30, 19.60, 20.70, and 19.60, respectively. The average values $\bar{c}_{0 \text { avg }}$ in these four sets of performance tests are $35.58,35.60,35.52$, and 35.98 , respectively. It can be observed that the randomly generated initial populations in the four sets of performance tests are similar, which means that the influence of the randomness of the initial population on the IMOHGA can be ignored in the instance studies.

The maximum iteration is predefined as 300 to ensure that the proposed algorithm is able to find the optimal individuals. Figure 19 shows the variation of the iterations required to find the feasible individuals in the 10 runs of the IMOHGA. The average of the iterations required to find the feasible individuals is 140.6, and the maximum and 


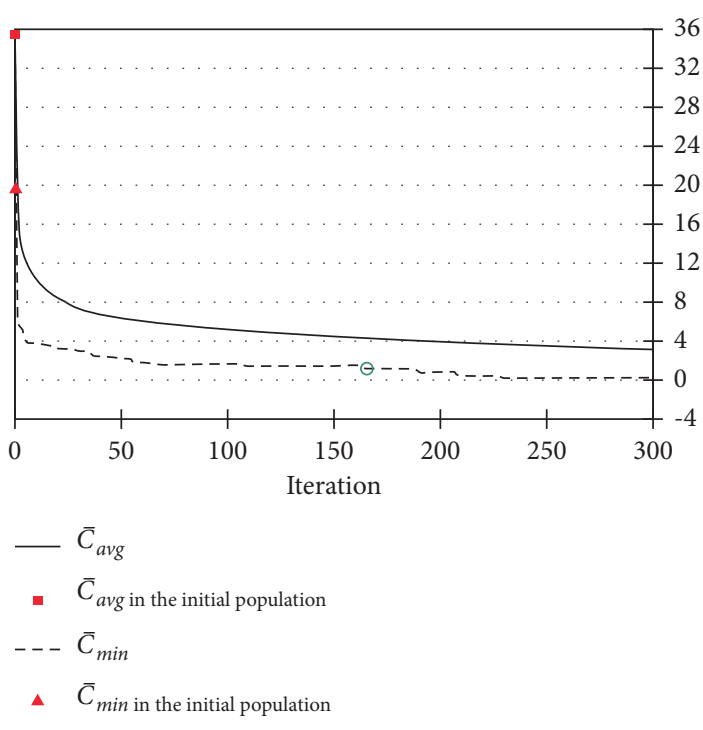

(a)

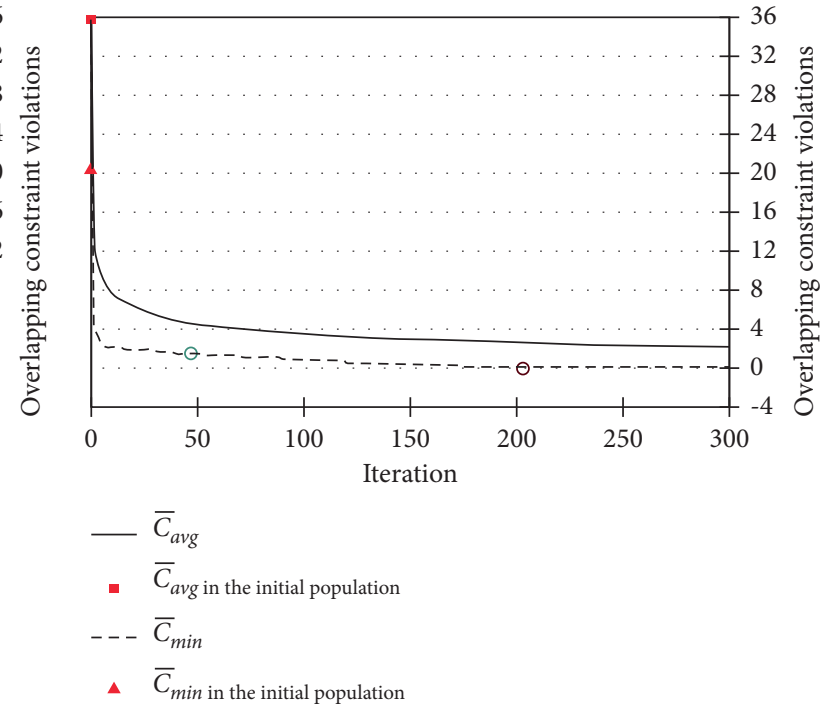

(b)

FIGURE 16: The influence of different crossover operators on performance indicators, $\bar{c}_{\text {avg }}$ and $\bar{c}_{\text {min }}$. (a) Using the multiple-point crossover operator. (b) Using the simulated binary crossover operator.

TABLE 4: Remarks of the optimization results by ship designers.

\begin{tabular}{lcccccc}
\hline & Figure 11(a) & Figure 11(b) & Figure 11(c) & Figure 11(d) & Figure 11(e) & Figure 11(f) \\
\hline 1st aspect & Good & Bad & Normal & Good & Good & Good \\
2nd aspect & Normal & Normal & Bad & Good & Normal & Good \\
3rd aspect & Good & Good & Bad & Good & Good & Bad \\
4th aspect & Bad & Normal & Normal & Normal & Bad & Normal \\
\hline
\end{tabular}

minimum of that are 90 and 253, respectively. This means that it is big enough to predefine the maximum iterations as 300 for the specific instance. However, the iteration range of 90 to 253 is a very wide interval, which indicates that the search process of the IMOHGA may have strong randomness.

5.3. The Behavior of the Proposed Algorithm. The proposed algorithm is designed to solve the specific problem. Its versatility needs to be verified, so the proposed algorithm is only compared with the standard NSGA-II in this paper. The results of the validation tests show that the standard NSGAII cannot find feasible solutions for the specific instance. As shown in Figure 9(b), when the nonlinear constraint violation of the individuals is reduced to 12 , it may be hard for the standard NSGA-II to further reduce its nonlinear constraint violation. Figure 9(a) shows that the IMOHGA finds the individuals with only one nonlinear constraint violation through 23 iterations and then finds the feasible solutions after continuing 77 iterations. This means that, as the number of nonlinear constraint violations decreases, the ability of the IMOHGA and standard NSGA-II to handle the nonlinear constraints is reduced, and the reduction of the standard NSGA-II is more significant.

Compared to the IMOHGA, the conventional algorithms, such as MOGA and NSGA-II, usually produce a lot of more nondominated solutions. The reason is that the topological replace operator used in the IMOHGA may eliminate many nondominated solutions. Unlike the conventional optimization problems, the purpose of the IMOHGA is not to find the uniformly spread out Paretooptimal front but to generate a set of diverse and rational arrangements. The requirement of diversity in the ship arrangement optimization problem is much stricter than that in the conventional optimization problems. Moreover, generating excessive nondominated solutions should be avoided in the ship arrangement optimization, because it may be a challenging and tedious task for a human to determine the best arrangements from a lot of nondominated solutions.

Figure 16 illustrates the behaviors of the simulated binary crossover and multiple-point crossover operators in handling nonlinear constraints. It can be observed that, with the assistance of the local search strategy, both the simulated binary crossover operator and the multiple-point crossover operator show excellent capabilities in handling the nonlinear constraints during the early iteration. However, as the number of nonlinear constraint violations is reduced to a low level, the simulated binary crossover operators would be significantly better than the multiple-point crossover operator in handling the nonlinear constraints. When the simulated binary crossover operator is used in the IMOHGA, the minimum and maximum of the iterations required to find the feasible individuals are 47 and 203, and 


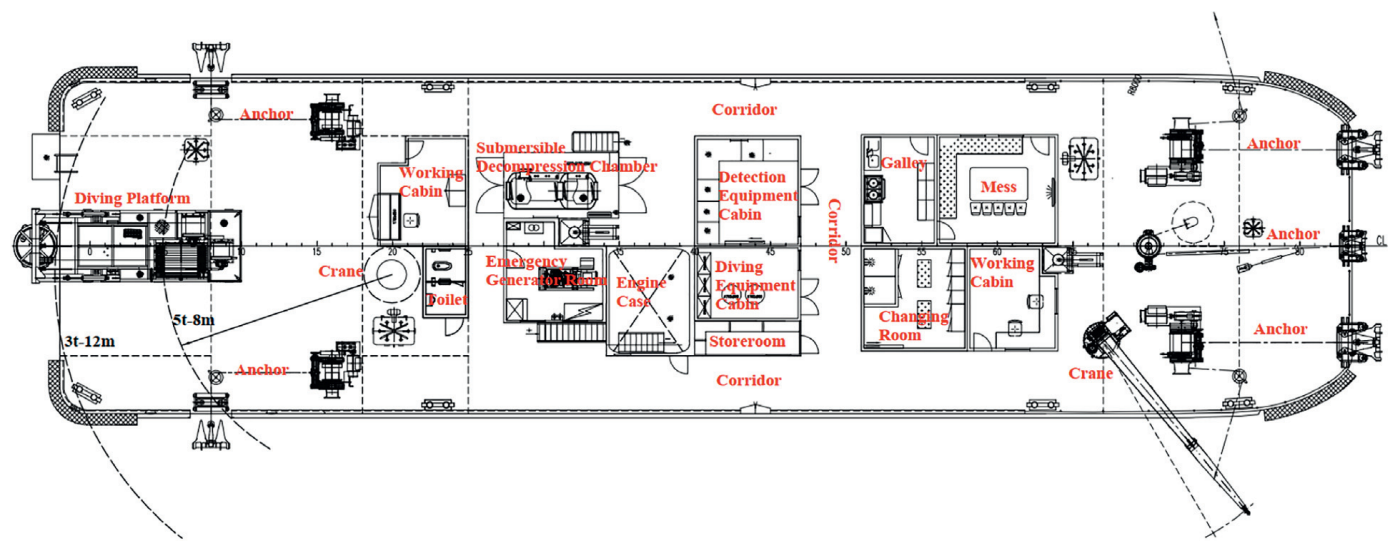

(a)

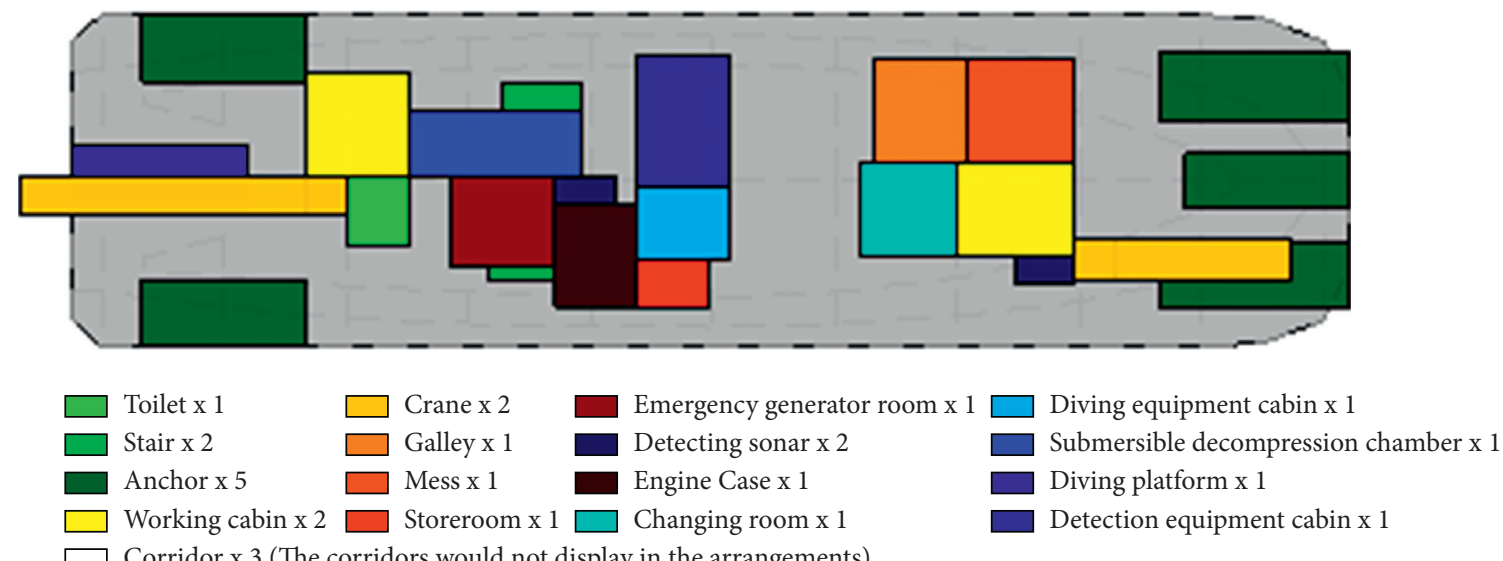

(b)

Figure 17: The comparison of the deck arrangement plans. (a) The detailed deck arrangement plans of the real ship. (b) The deck arrangement plan generated by the proposed algorithm.

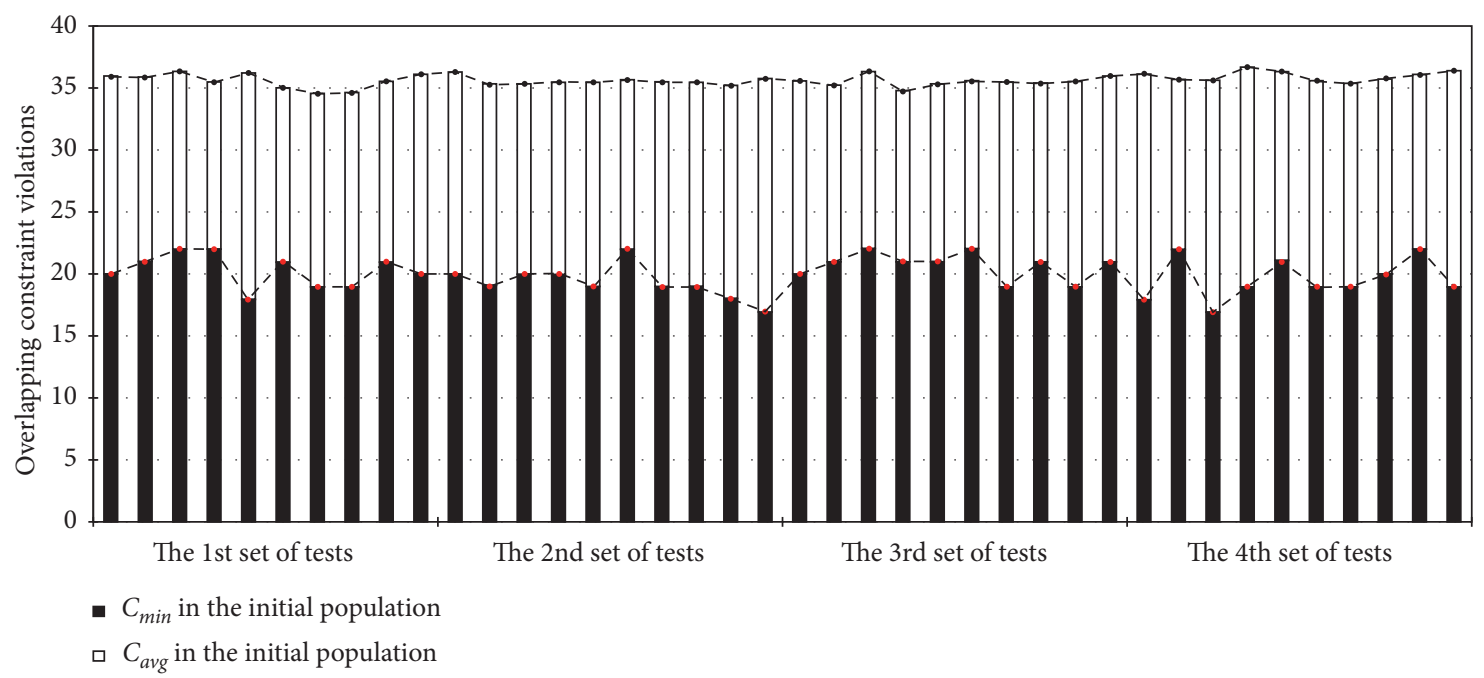

FIGURE 18: The minimum number and the average number of overlapping constraint violations in the initial population.

when the multiple-point crossover operator is used, the minimum of the iterations required to find the feasible individuals is more than 150 and the maximum of that is more than 300 , which means that the feasible individuals may not be found until the iteration is terminated. Figure 13 illustrates the behaviors of the adaptive crossover operator in handling nonlinear constraints. The capability of the adaptive crossover operator in handling overlapping 


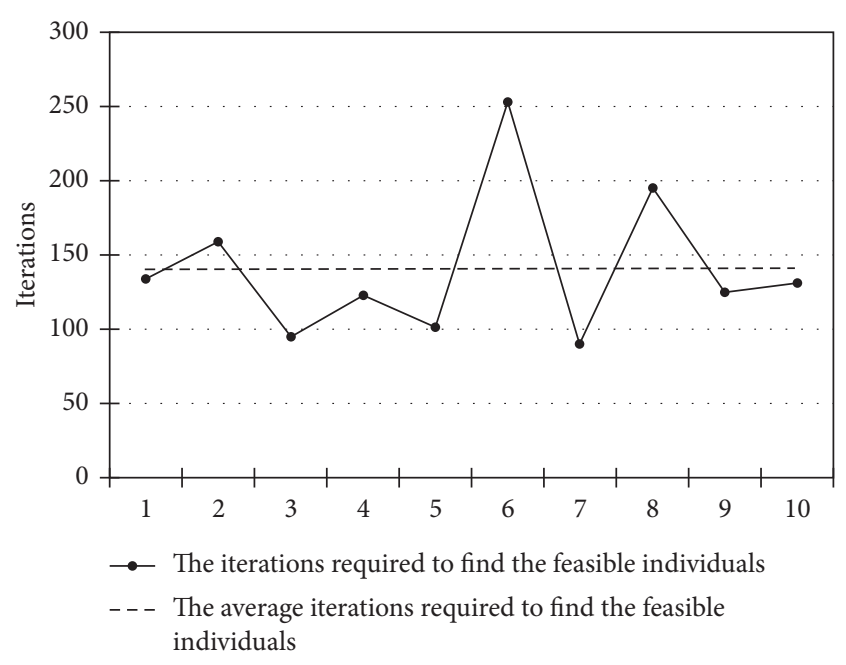

Figure 19: The variation of the iterations required to find the feasible individuals.

constraints is slightly worse than the simulated binary crossover operator, in which the adaptive crossover operator requires nearly 50 more iterations than the simulated binary crossover operator to find the feasible individuals. However, the capability of the adaptive crossover operator in handling the overlapping constraints is still enough and acceptable for the specific instance. Furthermore, the box plot (Figure 10) can demonstrate that, using the adaptive crossover operator, the nonlinear constraint violations of the population distribute under 6, and those of the Pareto-optimal group usually reach zero. This confirms the ability of the IMOHGA to generate feasible deck arrangements for the instance.

Figure 14 compares the capability of the three crossover operators in maintaining the diversity of population with the assistance of the topological replace operator. Although all three crossover operators drastically reduce the diversity of population during the early iteration, they can maintain the diversity of the Pareto-optimal group at a good level. The adaptive crossover operator and the multiple-point crossover operator would generate the Pareto-optimal group with a diversity of 0.9 , which are better than the simulated binary crossover operator. Figure 15 shows the effect of the topological replace operator in maintaining the diversity of population and Pareto-optimal group. It can be observed that, without the use of the topological replace operator, the diversity of the population would drop sharply to around 0 , which means that the deck arrangements generated are almost identical. Therefore, the traditional diversity protection strategies used in NSGA-II are ineffective for the ship arrangement optimization problem, and it is necessary to design a novel diversity protection strategy for these specific problems. The topological replace operator proposed in this paper may be a meaningful attempt and has achieved good results.

\section{Conclusion}

The ship general arrangement optimization problem is essential and difficult. During the last decades, numerous attempts have been done and several optimization methods have been developed. However, despite many efforts, it is still impossible to solve the optimization problem at the level of ship general arrangement design. The literature review presented in Section 1 indicates that these approaches are only available for the ship compartment (Zone-deck) arrangement design or generate feasible ship arrangements at the geometrical level or topological level. This paper proposed an IMOHGA to solve the ship deck arrangement optimization. The arrangement space in the deck arrangement optimization is much bigger than that in the ship compartment (Zone-deck) arrangement optimization, and much more arranged objects would be taken into consideration in the ship deck arrangement optimization.

It is well known that the assessment of the ship arrangement design is very subjective and ambiguous work and involves many different aspects. To overcome these, the Fuzzy Set Theory is used in the proposed approach and the assessment is divided into topological assessment and geometric assessment. It may be possible to gather multiobjectives in two objective functions: the topological objective function and geometric objective function. And an overlapping objective is also used in the mathematical model as the third objective function to accelerate convergence. These objective functions fail to explain how good the generated arrangements are, but they provide an objective and consistent judgment for the generated deck arrangements.

This paper presented and discussed the test results of the IMOHGA in solving the main deck arrangement optimization of an underwater detection ship, where 72 optimization variables and 222 nonlinear constraints corresponding to 27 arranged objects were taken into consideration. The validation tests demonstrate that the proposed algorithm is successful in generating diverse deck arrangements for the highly constrained problem which the standard NSGA-II fails to solve. However, the generated deck arrangements cannot be directly used in the general arrangement design of ships. The main purpose is not to substitute the ship designers but to assist them in determining the ship general arrangement in the early stage of ship design. In the performance tests, the evolving behaviors of the nonlinear constraint violations, the analysis of robustness, and the comparison of three different crossover operators illustrate the performance of the IMOHGA. Furthermore, another important improvement of the IMOHGA is the fact that the diversity of the generated arrangements is preserved by using the novel topological replace operator.

A future research work of this approach would be to tackle the multideck arrangement optimization problem. A more complex method of connectivity distance calculation would be used, and the major structure boundaries would also be taken into consideration. This would make the generated arrangements closer in practice.

\section{Data Availability}

The optimization data used to support the findings of this study are included within the article. 


\section{Conflicts of Interest}

The authors declare that they have no conflicts of interest.

\section{Acknowledgments}

This work was supported by the Green Intelligent Inland Ship Innovation Programme.

\section{References}

[1] C. M. Carlson and D. R. Cebulski, "Computer-aided ship arrangement design," Naval Engineers Journal, vol. 86, no. 5, pp. 33-40, 1974.

[2] B. C. Nehrling, "Fuzzy set theory and general arrangement design," in Proceedings of the Fifth International Conference Compuer Applications in the Automation of Shipyard Operation and Ship Design, Trieste, Italy, September 1985.

[3] A. S. Daniels and M. G. Parsons, "An agent based approach to space allocation in general arrangements," in Proceedings of the 7th International Marine Design Conference, Ann Aobor, MI, USA, May 2006.

[4] M. G. Parsons, H. Chung, E. Nick, A. Daniels, S. Liu, and J. Patel, "Intelligent ship arrangements: a new approach to general arrangement," Naval Engineers Journal, vol. 120, no. 3, pp. 51-65, 2008.

[5] A. S. Daniels, F. Tahmasbi, and D. J. Singer, "Intelligent ship arrangement passage variable lattice network studies and results," Naval Engineers Journal, vol. 122, no. 2, pp. 107-119, 2010.

[6] A. S. Daniels and M. G. Parsons, "A hybrid agent - genetic algorithm approach to general arrangements," Ship Technology Research, vol. 55, no. 2, pp. 78-86, 2015.

[7] K.-Y. Lee, S.-N. Han, and M.-I. Roh, "An improved genetic algorithm for facility layout problems having inner structure walls and passages," Computers \& Operations Research, vol. 30, no. 1, pp. 117-138, 2003.

[8] K.-Y. Lee, M.-I. Roh, and H.-S. Jeong, “An improved genetic algorithm for multi-floor facility layout problems having inner structure walls and passages," Computers \& Operations Research, vol. 32, no. 4, pp. 879-899, 2005.

[9] E. K. Boulougouris, A. D. Papanikolaou, G. Z. Ntua-sdl, and G. Zaraphonitis, "Optimization of arrangements of ro-ro passenger ships with genetic algorithms," Ship Technology Research, vol. 51, pp. 99-105, 2003.

[10] A. İ. Ölçer, C. Tuzcu, and O. Turan, "An integrated multiobjective optimisation and fuzzy multi-attributive group decision-making technique for subdivision arrangement of Ro-Ro vessels," Applied Soft Computing, vol. 6, no. 3, pp. 221-243, 2006.

[11] A. İ. Ölçer, "A hybrid approach for multi-objective combinatorial optimisation problems in ship design and shipping," Computers \& Operations Research, vol. 35, no. 9, pp. 27602775, 2008.

[12] J. W. Gillespie, A Network Science Approach to Understanding and Generating Ship Arrangements in Early-Stage Design, University of Michigan, Michigan, MI, USA, 2012.

[13] J. W. Gillespie, A. S. Daniels, and D. J. Singer, "Generating functional complex-based ship arrangements using network partitioning and community preferences," Ocean Engineering, vol. 72, pp. 107-115, 2013.

[14] J. W. Gillespie and D. J. Singer, "Identifying drivers of general arrangements through the use of network measures of centrality and hierarchy," Ocean Engineering, vol. 57, pp. 230-239, 2013.

[15] B. Van Oers, D. Stapersma, and H. Hopman, "Development and implementation of an optimisation-based space allocation routine for the generation of feasible concept designs," in Proceedings of the Computer Applications and Information Technology in the Maritime Industries, pp. 171-185, Cortona, Italy, April 2007.

[16] B. Van Oers, D. Stapersma, and H. Hopman, "A 3D packing approach for the early stage configuration design of ships," in Proceedings of the International Naval Engineering Conference, pp. 367-381, Portsmouth, UK, May 2010.

[17] K. Wanger, A. Wassink, B. van Oers, and H. Hopman, "Modeling complex vessels for use in a 3D packing approach: an application to deepwater drilling vessel design," in Proceedings of the Computer Applications and Information Technology in the Maritime Industries, pp. 259-272, Gubbio, Italy, 2010.

[18] B. Van Oers, A Packing Approach for the Early Stage Design of Service Vessels, Delft University of Technology, Delft, Netherlands, 2011.

[19] B. Van Oers, D. Stapersma, and H. Hopman, "Issues when selecting naval ship configurations from a pareto-optimal set," in Proceedings of the 12th AIAA/ISSMO Multidisciplinary Analysis and Optimization Conference, Victoria, Australia, September 2008.

[20] B.-Y. Chung, S.-Y. Kim, S.-C. Shin, Y.-H. Koo, and A. Kraus, "Optimization of compartments arrangement of submarine pressure hull with knowledge based system," International Journal of Naval Architecture and Ocean Engineering, vol. 3, no. 4, pp. 254-262, September 2011.

[21] K.-S. Kim, M.-I. Roh, and S. Ha, "Expert system based on the arrangement evaluation model for the arrangement design of a submarine," Expert Systems with Applications, vol. 42, no. 22, pp. 8731-8744, 2015.

[22] K.-S. Kim and M.-I. Roh, "A submarine arrangement design program based on the expert system and the multistage optimization," Advances in Engineering Software, vol. 98, pp. 97-111, 2016.

[23] M. K. Thakur, M. Kumari, and M. Das, "Architectural layout planning using Genetic Algorithms," in Proceedings of the 2010 3rd International Conference on Computer Science and Information Technology, July 2010.

[24] E. Rodrigues, A. R. Gaspar, and Á. Gomes, “An approach to the multi-level space allocation problem in architecture using a hybrid evolutionary technique," Automation in Construction, vol. 35, pp. 482-498, 2013.

[25] E. Rodrigues, A. R. Gaspar, and Á. Gomes, “An evolutionary strategy enhanced with a local search technique for the space allocation problem in architecture, Part 2: validation and performance tests," Computer-Aided Design, vol. 45, no. 5, pp. 898-910, 2013.

[26] E. Rodrigues, A. R. Gaspar, and Á. Gomes, “An evolutionary strategy enhanced with a local search technique for the space allocation problem in architecture, Part 1: Methodology," Computer-Aided Design, vol. 45, no. 5, pp. 887-897, 2013.

[27] E. Rodrigues, A. R. Amaral, A. R. Gaspar, and Á. Gomes, “An approach to urban quarter design using building generative design and thermal performance optimization," Energy Procedia, vol. 78, pp. 2899-2904, 2015.

[28] I.-C. Yeh, "Architectural layout optimization using annealed neural network," Automation in Construction, vol. 15, no. 4, pp. 531-539, 2006. 
[29] Z. X. Liang, L. Yan, and J. Z. shang, "Ship cabin layout design using game theory," Journal of Marine Science and Technology, vol. 13, no. 4, pp. 446-454, 2008.

[30] J. Bénabès, F. Bennis, E. Poirson, and Y. Ravaut, "An interactive-based approach to the layout design optimization," Global Product Development, pp. 511-520, 2011.

[31] M. Inoue and H. Takagi, "Layout algorithm for an EC-based room layout planning support system," in Proceedings of the 2008 IEEE Conference on Soft Computing in Industrial Applications, Muroran, Japan, 2008.

[32] E. K. Nick, Fuzzy Optimal Allocation and Arrangement of Spaces in Naval Surface Ship Design, University of Michigan, Michigan, MI, USA, 2008.

[33] K. Deb, A. Pratap, S. Agarwal, and T. Meyarivan, "A fast and elitist multiobjective genetic algorithm: NSGA-II," IEEE Transactions on Evolutionary Computation, vol. 6, no. 2, pp. 182-197, 2002.

[34] H. Wang, S. Chen, and L. Luo, "An elitist non-dominated sorting hybrid evolutionary algorithm for multi-objective constrained ship arrangements optimization problem," Communications in Computer and Information Science, vol. 791, pp. 52-67, 2017. 